\title{
Unification and Evaluation of Equalization Structures and Design Algorithms for Discrete Multitone Modulation Systems
}

\author{
Richard K. Martin \\ Air Force Institute of Technology, AFIT/ENG \\ Dept. of Electrical \& Computer Engineering \\ Wright-Patt AFB, OH 45433-7765, USA \\ richard.martineafit.edu \\ Ming Ding \\ Bandspeed, Inc. \\ 4301 Westbank Drive, Bldg. B, Suite 100 \\ Austin, TX 78746, USA \\ mding@bandspeed.com \\ Milos Milosevic \\ Schlumberger \\ Sugar Land, TX 77478, USA \\ mmiloseviceaustin.rr.com \\ Marc Moonen \\ ESAT-SISTA K.U. Leuven \\ Kasteelpark Arenberg 10, Office: 01.69 \\ B-3001 Leuven-Heverlee, Belgium \\ Marc.Moonendesat.kuleuven.ac.be
}

\author{
Koen Vanbleu \\ Broadcom UK Ltd. \\ 2800 Mechelen, Belgium \\ koen. vanbleu@telenet.be
}

\author{
Geert Ysebaert \\ Alcatel Telecom - Research and Innovation \\ Francis Wellesplein, 1 \\ 2018 Antwerpen, Belgium \\ geert.ysebaertealcatel.be \\ Brian L. Evans \\ The University of Texas at Austin \\ Dept. of Electrical \& Computer Engineering \\ Engineering Science Building, Room 433B \\ 1 University Station C0803 \\ Austin, TX 78712-1084, USA \\ bevans@ece.utexas.edu \\ C. Richard Johnson, Jr. \\ Cornell University, 390 Rhodes Hall \\ School of Electrical \& Computer Engineering \\ Ithaca, NY 14853, USA \\ johnsondece.cornell.edu
}

(C) 2004 IEEE. To appear in IEEE Transactions on Signal Processing, approximately summer 2005. Personal use of this material is permitted. However, permission to reprint/republish this material for advertising or promotional purposes or for creating new collective works for resale or redistribution to servers or lists, or to reuse any copyrighted component of this work in other works, must be obtained from the IEEE. Contact:

IEEE Intellectual Property Rights Office

IEEE Service Center

445 Hoes Lane

P.O. Box 1331

Piscataway, NJ 08855-1331 USA

Telephone: (732) 562-3966

Fax: (732) 981-8062

copyrights@ieee.org

http://www.ieee.org 


\title{
Unification and Evaluation of Equalization Structures and Design Algorithms for Discrete Multitone Modulation Systems
}

\author{
Richard K. Martin, Member, IEEE, Koen Vanbleu, Member, IEEE, Ming Ding, Member, IEEE, \\ Geert Ysebaert, Member, IEEE, Milos Milosevic, Member, IEEE, Brian L. Evans, Senior Member, IEEE, \\ Marc Moonen, Member, IEEE, C. Richard Johnson, Jr., Fellow, IEEE
}

\begin{abstract}
To ease equalization in a multicarrier system, a cyclic prefix $(\mathrm{CP})$ is typically inserted between successive symbols. When the channel order exceeds the $\mathrm{CP}$ length, equalization can be accomplished via a time-domain equalizer (TEQ), which is a finite impulse response (FIR) filter. The TEQ is placed in cascade with the channel to produce an effective shortened impulse response. Alternatively, a bank of equalizers can remove the interference tone-by-tone. This paper presents a unified treatment of equalizer designs for multicarrier receivers, with an emphasis on discrete multitone systems. It is shown that almost all equalizer designs share a common mathematical framework based on the maximization of a product of generalized Rayleigh quotients. This framework is used to give an overview of existing designs (including an extensive literature survey), to apply a unified notation, and to present various common strategies to obtain a solution. Moreover, the unification emphasizes the differences between the methods, enabling a comparison of their advantages and disadvantages. In addition, 16 different equalizer structures and design procedures are compared in terms of computational complexity and achievable bit rate using synthetic and measured data.
\end{abstract}

\section{INTRODUCTION TO MULTICARRIER EQUALIZATION}

During the last decade, extensive research has been done to provide broadband communication to and from the customer

Manuscript received December 14, 2003; revised June 3, 2004 and September 30, 2004. The associate editor coordinating the review of this paper and approving it for publication was Dr. Xiaodong Wang.

This work was supported in part by NSF grant CCR-0310023; Applied Signal Technology (Sunnyvale, CA); Texas Instruments (Dallas, TX); the Belgian State, Prime Minister's Office - Federal Office for Scientific, Technical and Cultural Affairs - Interuniversity Poles of Attraction Programme (2002-2007) - IUAP P5/22 and P5/11; the Concerted Research Action GOA-MEFISTO666 of the Flemish Government; Research Project FWO nr. G.0196.02; and The State of Texas Advanced Technology Program under project 0036580614-2001. The views expressed in this paper are those of the authors, and do not reflect the official policy or position of the United States Air Force, Department of Defense, or the U.S. Government. This document has been approved for public release; distribution unlimited. The scientific responsibility is assumed by the authors.

R. K. Martin is with the Dept. of Elec. and Comp. Eng., The Air Force Inst. of Technology, WPABF, OH 45433 (richard.martin@afit.edu). M. Ding is with Bandspeed, Inc., Austin, TX (mding@bandspeed.com). K. Vanbleu is with Broadcom UK Ltd., Mechelen, Belgium (koen.vanbleu@telenet.be). M. Milosevic is with Schlumberger, Sugar Land, TX 77478 USA (mmilosevic@austin.rr.com). G. Ysebaert is with Alcatel Telecom, Antwerpen, Belgium (geert.ysebaert@alcatel.be). Brian Evans is with the Dept. of Elec. and Comp. Eng. at The University of Texas at Austin (bevans@ece.utexas.edu). M. Moonen is with the Katholieke Universiteit Leuven - ESAT-SCD/SISTA, 3001 Leuven-Heverlee, Belgium (moonen@esat.kuleuven.ac.be). C. R. Johnson, Jr., is with the School of Elec. and Comp. Eng., Cornell University, Ithaca, NY 14853 (johnson@ece.cornell.edu).

Digital Object Identifier xxxxxxxx premises. To cope with the time dispersive transmission characteristics of wireline and wireless communications, multicarrier (MC) modulation offers a viable solution. In the 1960s, the first MC systems were conceived and implemented [1], [2], albeit only in analog form. In 1971, a widespread interest was created due to an all-digital implementation based on the fast Fourier transform (FFT) [3]. Today, MC modulation is used in digital audio/video broadcasting [4], [5], in wireless local area networks [6], [7], and in digital subscriber lines (DSL) [8], [9], [10], [11].

The multicarrier system model is shown in Fig. 1. The binary input data stream is split into $N$ groups of bits, which are then passed through $N$ "constellation mappers" [commonly quadrature amplitude modulation (QAM)]. The $N$ complex-valued outputs are passed through an $N$-point inverse discrete Fourier transform (IDFT), implemented by an inverse FFT. After the signal is passed through a physical channel, the receiver uses a DFT to recover the data within a bit error rate tolerance.

MC systems based on discrete multitone (DMT) modulation as defined in asymmetric and very high speed DSL (ADSL, VDSL) standards and orthogonal frequency division multiplexing (OFDM) as defined in IEEE 802.11a and HIPERLAN2 standards use an elegant equalization method. A cyclic prefix (CP), consisting of a copy of the last $\nu$ samples of each symbol, is prepended to the IFFT output block before transmission [12], [13]. The resulting finitelength time-domain signal is the symbol to be transmitted. If $\nu \geq L_{h}$, where $L_{h}$ is the FIR channel order, the linear convolutive channel is converted to a circular one. The circulant convolution matrix is diagonalized by the IDFT and DFT matrices, so the transmitted data can be recovered by a bank of complex scalars, called a frequency-domain equalizer (FEQ). This channel order condition is often true for wireless OFDM [14]. When $L_{h}>\nu$, which is e.g. the case for ADSL modems, the convolution is no longer circular, which results in inter-symbol and inter-carrier interference (ISI, ICI) [15]. To mitigate this interference, a time-domain equalizer (TEQ), which is an FIR filter, can be introduced before the FFT.

The goal of TEQ design is application-dependent: in a wireless scenario, bit-error rate minimization and fast adaptation to nonstationary environments are desired; whereas in DSL, bit rate maximization in a quasi-stationary environment is targeted. This paper focuses on the latter case. Most TEQ 


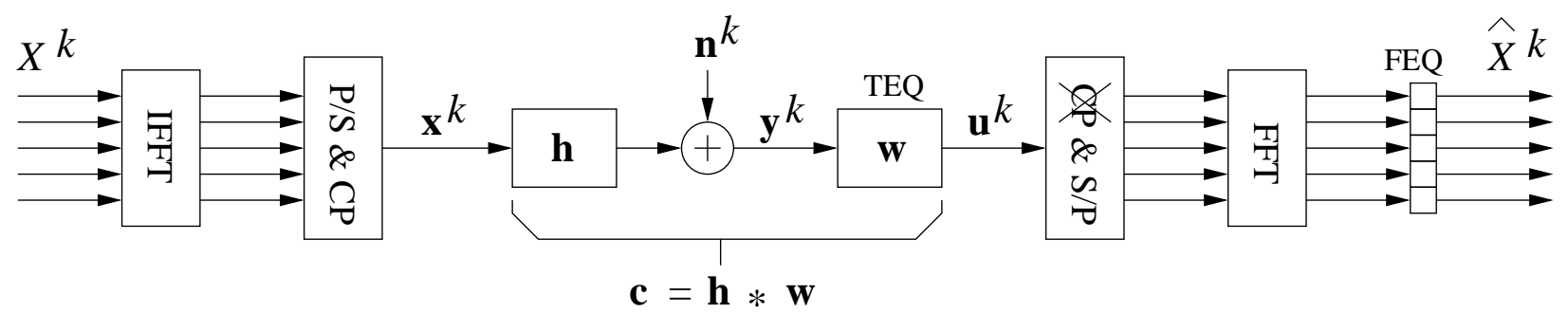

Fig. 1. Multicarrier system model. (I)FFT: (inverse) fast Fourier transform, P/S: parallel to serial, S/P: serial to parallel, CP: add cyclic prefix, and xCP: remove cyclic prefix.

designs have been proposed in the DSL context. TEQ design has inspired many researchers because bit rate optimization leads to a highly non-linear optimization problem. Hence, simplified procedures are resorted to, which are primarily based on time domain channel shortening (rather than bit rate maximization). Here, the TEQ is designed so that the convolution of the channel $\mathbf{h}$ (modeled as an FIR filter including transmit/receive front end filters and the physical transmission medium) and the TEQ $\mathbf{w}$ produces an overall impulse response with almost all of its energy concentrated in a length $\nu+1$ window.

The intriguing problems encountered in TEQ design are mainly due to the demodulation via the FFT. Since the TEQ is before the FFT, all frequency bins are treated in a combined fashion. Moreover, the poor spectral containment of the demodulating FFT imposes a difficult interference structure and may lead to noise enhancement combined with "noise pick-up" from out-of-band noise [16]. Alternatively, one could consider a bank of equalizers, one per subcarrier. This approach is a generalization of the TEQ, which means that its performance should be as good as or better than the optimal TEQ [17], [18]. An even more general receiver structure exists where the cascade of the FFT and this equalizer filterbank is replaced by a new set of parallel filters [19], [20], [21], which act directly on the time domain samples.

This paper presents an overview of the various equalizer designs. The goals are to provide a unified mathematical framework and a unified notation for different equalizer designs, an extensive literature survey, and an objective performance evaluation. Although channel shortening could be generalized to the multiple-input, multiple-output (MIMO) case [22], [23], [24], [25], [26], [27], we will restrict ourselves to the singleinput, single-output (SISO) case.

The paper is organized as follows. In Section II the unified TEQ design framework is formulated as a product of Rayleigh quotients. Optimal TEQ designs of this form are presented in Section III. Section IV presents simpler designs which use multiple filters that each maximize a single generalized Rayleigh quotient. Section V presents yet simpler designs which only require one filter that maximizes a single generalized Rayleigh quotient. Exceptions to the common formulation are treated in Section VI. Bit rate and complexity comparisons are given in Section VII, and Section VIII concludes. Throughout, the notation will be:

- $N$ is the (I)DFT size, $\nu$ is the prefix length, $s=N+\nu$ is the symbol size, $N_{u}$ is the number of used tones, $\mathcal{S}$ is the set of used tones, $i$ is the tone index, $k$ is the DMT symbol index, and $\Delta$ is the synchronization delay.

- $\mathcal{F}_{N}$ and $\mathcal{I}_{N}$ are the $N$-point DFT and IDFT matrices, respectively; $\mathbf{f}_{i}$ is the $i$-th DFT row.

- The transmitted frequency domain symbol vector at time $k$ is $X^{k}$; its $i$-th entry is $X_{i}^{k}$. The transmitted (timedomain), received, and TEQ output sequences are $x(l)$, $y(l)$, and $u(l)$, respectively. Vectors consisting of consecutive samples of the $k^{\text {th }}$ block of these sequences are $\mathbf{x}^{k}, \mathbf{y}^{k}$, and $\mathbf{u}^{k}$; their lengths and start indices depend on the design, hence these will be given in each section of the paper. Vectors are in bold with element numbers in brackets (e.g. $\mathbf{y}^{k}[l]$ ), and time-domain signals are in italics with the time index in parentheses (e.g. $y(s k+1)$ ).

- $\mathbf{w}, \mathbf{h}$, and $\mathbf{c}=\mathbf{h} \star \mathbf{w}$ are vectors containing the TEQ, channel, and effective channel impulse responses of orders $L_{w}, L_{h}$, and $L_{c}$, respectively, where $\star$ denotes linear convolution.

- $\mathbf{0}_{m \times n}$ is the all zero matrix of size $m \times n$; $\mathbf{I}_{n}$ is the identity matrix of size $n \times n$.

- $(\cdot)^{T},(\cdot)^{H},(\cdot)^{*}, \mathcal{E}\{\cdot\}$ denote transpose, Hermitian, complex conjugate, and expectation respectively.

\section{COMMON FORMULATION}

There are many ways of designing the DMT equalizer, depending on how the problem is posed. However, almost all of the algorithms fit into the same formulation: the maximization of a generalized Rayleigh quotient or a product of generalized Rayleigh quotients. Consider the optimization problem

$$
\widehat{\mathbf{w}}^{o p t}=\arg \max _{\widehat{\mathbf{w}}} \prod_{j=1}^{M} \frac{\widehat{\mathbf{w}}^{T} \mathbf{B}_{j} \widehat{\mathbf{w}}}{\widehat{\mathbf{w}}^{T} \mathbf{A}_{j} \widehat{\mathbf{w}}}
$$

In general, the solution to (1) is not well-understood when $M>1$. However, for $M=1$,

$$
\widehat{\mathbf{w}}^{\text {opt }}=\arg \max _{\widehat{\mathbf{w}}} \frac{\widehat{\mathbf{w}}^{T} \mathbf{B} \widehat{\mathbf{w}}}{\widehat{\mathbf{w}}^{T} \mathbf{A} \widehat{\mathbf{w}}},
$$

the solution is the generalized eigenvector of the matrix pair $(\mathbf{B}, \mathbf{A})$ corresponding to the largest generalized eigenvalue [28]. Most TEQ designs fall into the category of (2), although several have $M \gg 1$ as in (1). The vector $\widehat{\mathbf{w}}$ to be optimized is usually the TEQ, but it may be e.g. the (shortened) target impulse response (TIR) [29], the per-tone equalizer [18], or half of a symmetric TEQ [30]. 
The solution to (2) requires computation of the $\widehat{\mathrm{w}}$ that satisfies [28]

$$
\mathbf{B} \widehat{\mathbf{w}}=\lambda \mathbf{A} \widehat{\mathbf{w}},
$$

where $\widehat{\mathrm{w}}$ corresponds to the largest generalized eigenvalue $\lambda$. If $\mathbf{A}$ is invertible, the problem can be reduced to finding an eigenvector of $\mathbf{A}^{-1} \mathbf{B}$. When $\mathbf{A}$ is symmetric, a more popular approach is to form the Cholesky decomposition $\mathbf{A}=\sqrt{\mathbf{A}} \sqrt{\mathbf{A}}^{T}$, and define $\hat{\mathbf{v}}=\sqrt{\mathbf{A}}^{T} \widehat{\mathbf{w}}$, as in [31]. Then

$$
\hat{\mathbf{v}}^{o p t}=\arg \max _{\hat{\mathbf{v}}} \frac{\hat{\mathbf{v}}^{T} \overbrace{\left(\sqrt{\mathbf{A}}^{-1} \mathbf{B} \sqrt{\mathbf{A}}-T\right)}^{\mathbf{C}} \hat{\mathbf{v}}}{\hat{\mathbf{v}}^{T} \hat{\mathbf{v}}} .
$$

The solution for $\hat{\mathbf{v}}$ is the eigenvector of $\mathbf{C}$ associated with the largest eigenvalue, and $\widehat{\mathbf{w}}=\sqrt{\mathbf{A}}^{-T} \hat{\mathbf{v}}$.

In some cases, $\mathbf{A}$ or $\mathbf{B}$ is the identity matrix, in which case (3) requires computation of a traditional eigenvector. There are many all-purpose eigenvector computation methods, such as the power method [28] and conjugate gradient methods [32]. More specific iterative eigensolvers may be designed for specific problems, such as the MERRY (Multicarrier Equalization by Restoration of RedundancY) algorithm [33] and Nafie and Gatherer's method [34], which iteratively compute the MSSNR TEQ [31].

The much more difficult case when $M>1$ in (1) is not well-understood. There may be many solutions that are locally but not necessarily globally optimal, so gradientdescent strategies only ensure convergence to a local optimum. One approach is to compute several reasonable initial guesses, apply gradient descent to each initialization, and then pick the best solution. This is not guaranteed to be optimal. The initial guesses can be made by computing the closed-form solutions for various $M=1$ cases, such as the MSSNR TEQ or TEQs that optimize the bit rate on individual tones [17].

The motivation for introducing the common framework of (1) is to show how almost all TEQ designs require similar solution techniques; and to show how the designs differ in terms of the number of generalized Rayleigh quotients $M$ and how the matrices $\mathbf{A}_{j}$ and $\mathbf{B}_{j}$ arise. The next section presents optimal designs that solve (1), and the following sections discuss approximate designs that solve (1).

\section{More than One RaYleigh QUOTIENT}

This section discusses TEQ designs that attempt to maximize the bit rate. All of the designs in this section can be cast in the form of (1) with $M>1$. The main distinctions between these designs are the approximations that are or are not made when modelling the SNR at the output for each tone. As we move through the section, we will include more and more approximations, hence we will be going in reverse historical order. We begin with a statement of the function to be optimized.

\section{A. Communications performance measure}

The performance metric adopted in this paper is the achievable bit rate for a fixed probability of error $\left(10^{-7}\right)$. Bit allocation on subcarrier $i$ is calculated by

$$
b_{i}=\left\lfloor\log _{2}\left(1+\frac{\mathrm{SNR}_{i}}{\Gamma_{\text {sim }}}\right)\right\rfloor
$$

where $\lfloor\cdot\rfloor$ is the flooring operation; $\mathrm{SNR}_{i}$ is the $\mathrm{SNR}$ at the $i$ th subcarrier, measured by averaging the output signal to noise (and residual interference) ratio at the FEQ output; and

$\Gamma_{\text {sim }}[\mathrm{dB}]=\Gamma_{\text {gap }}[\mathrm{dB}]+$ system margin $[\mathrm{dB}]-$ coding gain $[\mathrm{dB}]$.

We will ignore the flooring function, as is standard practice [35], [36]. The "SNR gap" $\Gamma_{g a p}=9.8 \mathrm{~dB}$ corresponds to $10^{-7}$ bit error rate, the system margin is $6 \mathrm{~dB}$, and the coding gain is $5 \mathrm{~dB}$ [11]. The achievable bit rate is then $R=f_{\text {sym }} \sum_{i} b_{i}$, where $f_{\text {sym }}=4 \mathrm{kHz}$ is the symbol rate and $b_{\mathrm{DMT}}=\sum_{i} b_{i}$ is the number of bits per DMT symbol. We will attempt to model the subchannel SNR as a generalized Rayleigh quotient,

$$
\mathrm{SNR}_{i}=\frac{\mathbf{w}^{T} \tilde{\mathbf{B}}_{i} \mathbf{w}}{\mathbf{w}^{T} \tilde{\mathbf{A}}_{i} \mathbf{w}} .
$$

Summing over $\mathcal{S}$, the set of $N_{u}$ subchannels that carry data, leads to a bit allocation of

$$
\begin{aligned}
b_{\mathrm{DMT}}(\mathbf{w}) & =\sum_{i \in \mathcal{S}} \log _{2}\left(1+\frac{\mathrm{SNR}_{i}}{\Gamma_{i}}\right) \\
& =\sum_{i \in \mathcal{S}} \log _{2} \frac{\mathbf{w}^{T}\left(\Gamma_{i} \tilde{\mathbf{A}}_{i}+\tilde{\mathbf{B}}_{i}\right) \mathbf{w}}{\mathbf{w}^{T}\left(\Gamma_{i} \tilde{\mathbf{A}}_{i}\right) \mathbf{w}} \\
& =\log _{2}\left(\prod_{i \in \mathcal{S}} \frac{\mathbf{w}^{T} \mathbf{B}_{i} \mathbf{w}}{\mathbf{w}^{T} \mathbf{A}_{i} \mathbf{w}}\right)
\end{aligned}
$$

Here, $\mathbf{B}_{i}=\Gamma_{i} \tilde{\mathbf{A}}_{i}+\tilde{\mathbf{B}}_{i}$ and $\mathbf{A}_{i}=\Gamma_{i} \tilde{\mathbf{A}}_{i}$. Maximizing (9) requires solving (1). The rest of this section presents the $\mathbf{A}_{i}$ and $\mathbf{B}_{i}$ matrices proposed as models by various researchers.

\section{B. Bitrate maximizing TEQ (BM-TEQ)}

Vanbleu et al. [37], [38] propose an exact subchannel SNR model defined at the FEQ output by exploiting the dependence of the FEQs on the TEQ coefficients. The resulting SNR model is a nonlinear function of the TEQ coefficients and accounts for the function of the FEQ as well.

Let $\mathbf{y}^{k}=\left[y\left(s k+\nu-L_{w}+1\right), \cdots, y(s(k+1))\right]^{T}$ be a vector of received samples of the current symbol $k$, and let $\mathbf{M}^{k}$ be an $N \times\left(L_{w}+1\right)$ Toeplitz matrix of elements of $\mathbf{y}^{k}[l]$,

$$
\mathbf{M}^{k}=\left[\begin{array}{ccc}
\mathbf{y}^{k}\left[L_{w}\right] & \cdots & \mathbf{y}^{k}[0] \\
\vdots & \ddots & \vdots \\
\mathbf{y}^{k}\left[L_{w}+N-1\right] & \cdots & \mathbf{y}^{k}[N-1]
\end{array}\right] .
$$

so that the FEQ input is given as the $i$-th FFT coefficient of y convolved with $\mathbf{w}$,

$$
U_{i}^{k}=\mathbf{f}_{i}\left(\mathbf{M}^{k} \mathbf{w}\right) .
$$

Then the FEQ output is given by

$$
D_{i} U_{i}^{k}=\alpha_{i} X_{i}^{k}+E_{i}^{k}
$$


where $D_{i}$ is the FEQ coefficient for tone $i, \alpha_{i}$ is a bias, due to the equalizer, and $E_{i}^{k}$ is the noise remaining on tone $i$. We assume unbiased MMSE FEQs,

$$
D_{i}=\frac{\mathcal{E}\left\{\left|X_{i}^{k}\right|^{2}\right\}}{\mathcal{E}\left\{\left(X_{i}^{k}\right)^{*} U_{i}^{k}\right\}}
$$

hence $\alpha_{i}$ is 1 and $E_{i}^{k}$ contains all noise sources, including residual ISI/ICI and crosstalk. The dependence of the FEQs on the TEQ leads to the subchannel SNR model

$$
S N R_{i}=\frac{\mathcal{E}\left\{\left|X_{i}^{k}\right|^{2}\right\}}{\mathcal{E}\left\{\left|D_{i} U_{i}^{k}-X_{i}^{k}\right|^{2}\right\}}=\frac{1}{\rho_{i}^{-2}-1}
$$

where

$$
\rho_{i}^{2}=\frac{\left|\mathcal{E}\left\{\left(X_{i}^{k}\right)^{*} U_{i}^{k}\right\}\right|^{2}}{\mathcal{E}\left\{\left|X_{i}^{k}\right|^{2}\right\} \mathcal{E}\left\{\left|U_{i}^{k}\right|^{2}\right\}} .
$$

Substituting (14) into the bit rate equation (8) and exploiting the model of the FEQ input (11), we obtain the form of (9) with

$$
\begin{aligned}
\mathbf{A}_{i}= & \Gamma_{i}\left(\mathcal{E}\left\{\left|X_{i}^{k}\right|^{2}\right\} \mathcal{E}\left\{\left(\mathbf{M}^{k}\right)^{H} \mathbf{f}_{i}^{H} \mathbf{f}_{i} \mathbf{M}^{k}\right\}\right. \\
& \left.-\mathcal{E}\left\{\left(\mathbf{M}^{k}\right)^{H} \mathbf{f}_{i}^{H} X_{i}^{k}\right\} \mathcal{E}\left\{\left(X_{i}^{k}\right)^{H} \mathbf{f}_{i} \mathbf{M}^{k}\right\}\right) \\
\mathbf{B}_{i}= & \Gamma_{i} \mathcal{E}\left\{\left|X_{i}^{k}\right|^{2}\right\} \mathcal{E}\left\{\left(\mathbf{M}^{k}\right)^{H} \mathbf{f}_{i}^{H} \mathbf{f}_{i} \mathbf{M}^{k}\right\}+ \\
& \left(1-\Gamma_{i}\right) \mathcal{E}\left\{\left(\mathbf{M}^{k}\right)^{H} \mathbf{f}_{i}^{H} X_{i}^{k}\right\} \mathcal{E}\left\{\left(X_{i}^{k}\right)^{H} \mathbf{f}_{i} \mathbf{M}^{k}\right\}
\end{aligned}
$$

Since this model is based on statistical expectations, it can be made arbitrarily accurate by averaging the empirical estimates of the expectations over enough data. However, the expectations can be costly to estimate. The remaining algorithms in this section use channel models to calculate $\mathbf{A}_{i}$ and $\mathbf{B}_{i}$.

Vanbleu, et al. propose maximizing the bit rate as a function of (16) and (17) by performing a gradient ascent of (9). Although this is not guaranteed to converge, good results have been reported [37].

\section{Maximum data rate (MDR) TEQ}

Milosevic et al. [17] proposed a design similar to the BMTEQ. The difference is that they explicitly model the nearend crosstalk, AWGN, analog-to-digital converter quantization noise, and the digital noise floor due to finite precision arithmetic, rather than considering all of these effects through expectations of various signals. Milosevic et al. write the subchannel SNR as a generalized Rayleigh quotient as in (7), with

$$
\begin{aligned}
& \tilde{\mathbf{A}}_{i}=2 S_{x, i}\left(\mathbf{H}_{\text {wall }, 1}^{T} \mathbf{V}_{i} \mathbf{V}_{i}^{H} \mathbf{H}_{\text {wall }, 1}+\mathbf{H}_{\text {wall }, 2}^{T} \mathbf{W}_{i} \mathbf{W}_{i}^{H} \mathbf{H}_{\text {wall }, 2}\right) \\
&+\mathbf{Q}_{i}^{\text {noise }} \mathbf{R}_{n}\left[\mathbf{Q}_{i}^{\text {noise }}\right]^{H}+\frac{\sigma_{\text {DNF }}^{2}}{\mathbf{w}^{T} \mathbf{w}} \mathbf{I}_{L_{w}+1}, \\
& \tilde{\mathbf{B}}_{i}=S_{x, i} \mathbf{H}^{T} \mathbf{Q}_{i}^{\text {circ }}\left[\mathbf{Q}_{i}^{\text {circ }}\right]^{H} \mathbf{H} .
\end{aligned}
$$

Here, $\mathbf{H}^{T}=\left[\mathbf{H}_{\text {wall }, 1}^{T}, \mathbf{H}_{\text {win }}^{T}, \mathbf{H}_{\text {wall, }, 2}^{T}\right]$ partitions the channel convolution matrix into signal (window) and ICI (wall) portions as in [31]; $\mathbf{V}_{i}$ and $\mathbf{W}_{i}$ are upper and lower triangular Hankel matrices made from the $i$ th row of the DFT matrix, $\mathbf{f}_{i}$; $\mathbf{Q}_{i}^{\text {noise }}$ and $\mathbf{Q}_{i}^{\text {circ }}$ are Hankel matrices made from $\mathbf{f}_{i} ; \mathbf{R}_{n}$ is the noise (AWGN and crosstalk) covariance matrix; and $\sigma_{\mathrm{DNF}}^{2}$ is the power of the noise due to the digital noise floor. See [17] for full definitions, although the partitioning of the channel convolution matrix will be treated in detail in Section V-B. The constraint $\mathbf{w}^{T} \mathbf{w}=1$ is used in [17] to remove the dependence of the last term of $\tilde{\mathbf{A}}$ on $\mathbf{w}$.

Sum-of-ratios maximization is an active research topic in the fractional programming community for which no definitive solution exists yet (see e.g. [39], [40]). However, the bit allocation (8) is a sum of logarithms of ratios, or a $\log$ of a product of ratios as in (1), thus maximizing it is an even more involved problem that of maximizing a sum of ratios. Milosevic et al. [17] use modifications of Almogy and Levin's approach to the sum-of-ratios problem [41] to optimize (8).

\section{Maximum bit rate $(M B R)$ method}

Arslan, Evans, and Kiaei [35], [36] proposed the Maximum Bit Rate (MBR) TEQ design, which follows the methods of separating channel impulse response into signal and interference paths (or "window" and "wall" portions) of [31]. The sub-channel SNR can be written as

$$
\mathrm{SNR}_{i}=\frac{S_{x, i}\left|C_{\text {signal }, i}\right|^{2}}{S_{n, i}\left|C_{\text {noise }, i}\right|^{2}+S_{x, i}\left|C_{I S I, i}\right|^{2}},
$$

where $S_{x, i}, S_{n, i}, C_{\text {signal }, i}, C_{n o i s e, i}$ and $C_{I S I, i}$ are the transmitted signal power, channel noise power, signal path gain, noise path gain, and the ISI path gain in the $i$ th sub-channel, modelled as

$$
\begin{aligned}
C_{\text {signal }, i} & =\mathbf{f}_{i} \operatorname{diag}(\mathbf{g}) \mathbf{H} \mathbf{w}, \\
C_{I S I, i} & =\mathbf{f}_{i}\left(\mathbf{I}_{N}-\operatorname{diag}(\mathbf{g})\right) \mathbf{H} \mathbf{w} \triangleq \mathbf{f}_{i} \mathbf{D} \mathbf{H} \mathbf{w}, \\
C_{\text {noise }, i} & =\mathbf{f}_{i}\left[\mathbf{w}^{T}, \quad \mathbf{0}_{1 \times\left(N-L_{w}-1\right)}\right]^{T} .
\end{aligned}
$$

Here, the $N \times 1$ vector $\mathbf{g}$ and the $N \times\left(L_{w}+1\right)$ convolution matrix $\mathbf{H}$ are given by

$$
\begin{aligned}
\mathbf{g}[n] & =\left\{\begin{array}{cc}
1, & \Delta \leq n \leq \Delta+\nu \\
0, & \text { otherwise }
\end{array}\right. \\
\mathbf{H} & =\left[\begin{array}{lll}
\mathbf{H}_{\text {wall }, 1}^{T}, & \mathbf{H}_{\text {win }}^{T}, & \mathbf{H}_{\text {wall }, 2}^{T}
\end{array}\right]^{T} .
\end{aligned}
$$

This leads to a subchannel SNR model as in (7) with

$$
\begin{aligned}
\widetilde{\mathbf{A}} & =S_{n, i} \mathbf{f}_{i}^{H}\left[0: L_{w}\right] \mathbf{f}_{i}\left[0: L_{w}\right]+S_{x} \mathbf{H}^{T} \mathbf{D} \mathbf{f}_{i}^{H} \mathbf{f}_{i} \mathbf{D H} \\
\widetilde{\mathbf{B}} & =S_{x} \mathbf{H}^{T} \operatorname{diag}(\mathbf{g}) \mathbf{f}_{i}^{H}\left[0: L_{w}\right] \mathbf{f}_{i}\left[0: L_{w}\right] \operatorname{diag}(\mathbf{g}) \mathbf{H} .
\end{aligned}
$$

Then the bit rate can be modelled as a sum of logs of generalized Rayleigh quotients, proceeding as in (8) to obtain $\mathbf{A}_{i}=\Gamma_{i} \tilde{\mathbf{A}}_{i}$ and $\mathbf{B}_{i}=\Gamma_{i} \tilde{\mathbf{A}}_{i}+\tilde{\mathbf{B}}_{i}$. Compared to the BM-TEQ and MDR methods, the model of the noise and interference is less precise and does not consider the effect of the digital noise floor or finite word-length effects. However, the model itself is somewhat easier to compute.

Arslan, et al. state that computing the MBR TEQ is not cost effective for a real-time system, and they proceed to approximate the MBR design by the Min-ISI design. The Min-ISI design requires maximization of a single generalized Rayleigh quotient, and it will be discussed in detail in Section V-D. 


\section{E. Maximum geometric signal-to-noise ratio (MGSNR) method}

Al-Dhahir and Cioffi [42], [43], [44], were the first to attempt bit rate maximization. Their approach was based on maximizing the geometric SNR (GSNR), which is approximately the geometric mean of the subchannel SNRs. Let $B_{i}$, $W_{i}$, and $H_{i}$ be the complex-valued frequency domain representations in subchannel $i$ of the (shortened) target impulse response (TIR) $\mathbf{b}$, the TEQ $\mathbf{w}$, and the transmission channel $\mathbf{h}$, respectively. Then the SNR in subchannel $i$, assuming equal signal power distribution in all subchannels, can be modelled as

$$
\begin{aligned}
\mathrm{SNR}_{i} & =\frac{S_{x}\left|H_{i}\right|^{2}}{S_{n, i}}=\frac{S_{x}\left|H_{i}\right|^{2}\left|W_{i}\right|^{2}}{S_{n, i}\left|W_{i}\right|^{2}} \\
\cong & \frac{S_{x}\left|B_{i}\right|^{2}}{S_{n, i}\left|W_{i}\right|^{2}}
\end{aligned}
$$

where $S_{x}$ and $S_{n, i}$ are the signal and noise powers in subchannel $i$, respectively. The GSNR is defined as

$$
\begin{aligned}
\mathrm{SNR}_{\text {geom }} & \triangleq \Gamma\left\{\left[\prod_{i \in \mathcal{S}}\left(1+\frac{\mathrm{SNR}_{i}}{\Gamma}\right)\right]^{\frac{1}{N_{u}}}-1\right\} \\
& \cong \prod_{i \in \mathcal{S}}\left(\mathrm{SNR}_{i}\right)^{\frac{1}{N_{u}}} \\
& \cong S_{x}\left[\prod_{i \in \mathcal{S}}\left(\frac{\left|B_{i}\right|^{2}}{S_{n, i}\left|W_{i}\right|^{2}}\right)\right]^{\frac{1}{N_{u}}}
\end{aligned}
$$

Here, $N_{u}$ is the size of the set of used carriers, $\mathcal{S}$. Several simplifying assumptions were made. It was assumed that $S N R_{i} \gg \Gamma$ for all $i$, so that the unity terms in (29) can be ignored. However, this is not true in subchannels with low SNR. Also, the subchannel SNR definition does not include the effects of the ISI, ICI, and DFT leakage in the denominator, but instead only the power of the noise after the equalizer. The model of the subchannel SNR (28) also assumes that

$$
\mathbf{f}_{i}(\mathbf{w} \star \mathbf{h})=\mathbf{f}_{i} \mathbf{w} \mathbf{f}_{i} \mathbf{h}=W_{i} H_{i} \text { and } B_{i}=W_{i} H_{i},
$$

where $\star$ is time domain linear convolution and $\mathbf{f}_{i}$ is the $i$ th DFT row (assumed to be truncated to the length of $\mathbf{w}$ or h). Linear convolution of $\mathbf{h}$ and $\mathbf{w}$ may not be equal to their product in the frequency domain, and the difference appears as a noise source. These assumptions tend to design a TEQ that ignores the subchannels with lower SNR (which contain significant ISI and ICI that the TEQ ought to mitigate) in favor of the subchannels with higher SNR, which does not maximize the data rate [35].

Under these assumptions, using (8) and (29), the DMT bit rate is approximately given by

$$
b_{\mathrm{DMT}}(\mathbf{w})=N_{u} \log _{2}\left(1+\frac{\mathrm{SNR}_{\text {geom }}}{\Gamma}\right) .
$$

Maximizing (29) maximizes (31) since the logarithmic function is monotonically increasing. Maximizing (29) is approxi- mately equivalent to maximizing the log of its numerator [43],

$$
\begin{aligned}
L(\mathbf{b}) & =\frac{1}{N_{u}} \sum_{i \in \mathcal{S}} \ln \left|B_{i}\right|^{2} \\
& =\frac{1}{N_{u}} \sum_{i \in \mathcal{S}} \ln \left(\mathbf{b}^{T} \mathbf{G}_{i} \mathbf{b}\right),
\end{aligned}
$$

where $\mathbf{G}_{i}=\mathbf{g}_{i} \mathbf{g}_{i}^{H}$, and $\mathbf{g}_{i}^{H}$ consists of the first $\nu+1$ elements of $\mathbf{f}_{i}$, the $i$-th row of the DFT matrix. The independence of the noise and the TEQ $\mathbf{w}$ on $\mathbf{b}$ is assumed in (32), which is not correct as $\mathbf{w}$ is a function of $\mathbf{b}$.

The unit norm constraint is imposed on $\mathbf{b}$ in order to keep it finite; however, according to [43], it then follows that $\left|B_{i}\right|^{2}=$ 1 for each $i$. This leads to a zero forcing solution for the TEQ w. Zero forcing is not necessary in DMT since it uses a guard band. To avoid the zero forcing solution another constraint is imposed: the mean squared error (MSE) at the TEQ output needs to be less than some value $\mathrm{MSE}_{\max }$. The optimal TIR $\mathbf{b}$ in terms of the maximum geometric SNR algorithm is then found by

$$
\begin{aligned}
\mathbf{b}_{\mathrm{GSNR}}^{\text {opt }}=\arg \max _{\mathbf{b}} L(\mathbf{b}) & =\arg \max _{\mathbf{b}} \prod_{i \in \mathcal{S}} \mathbf{b}^{T} \mathbf{G}_{i} \mathbf{b} \\
\text { such that } \quad \mathbf{b}^{T} \mathbf{b} & =1 \\
\text { and } \mathbf{b}^{T} \mathbf{R}_{\Delta} \mathbf{b} & <\mathrm{MSE}_{\max },
\end{aligned}
$$

where $\mathbf{b}^{T} \mathbf{R}_{\Delta} \mathbf{b}$ is the MSE, and $\mathbf{R}_{\Delta}$ is the $\mathbf{A}$ matrix from the MMSE design, as will be discussed in Section V-A, equation (56). Note that (33) is equivalent to (1) with $\mathbf{B}_{i}=\mathbf{G}_{i}$ and $\mathbf{A}_{i}=\mathbf{I}_{\nu+1}$, but with an extra inequality constraint. Once the optimal TIR is found, the optimal TEQ $w$ is the one which produces this TIR when convolved with the channel. In [44], the the subchannel SNR model of (28) was modified to include partially the effects of the ISI, but only when evaluating the TEQ designed using (28).

Currently, this non-linear optimization problem can only be solved numerically. Al-Dhahir and Cioffi [43] use Matlab's non-linear optimization toolbox to compute the TIR, hence the MGSNR TEQ is not feasible for implementation on a real-time DSP. However, their approach was the first attempt to directly maximize the bit rate. An iterative GSNR maximization method was presented in [45].

\section{Multiple FILTERS, EACH WITH A SINGLE QUOTIENT}

This section also presents equalizer designs that maximize the DMT system bit rate. Whereas in the previous section a single time-domain equalizer was designed to equalize all frequency bins together in an optimal way, the idea here is that each data-carrying subchannel receives its own equalizer which is designed to maximize the bit rate on that subchannel. By extension, if every subchannel carries the maximum number of bits, then the bit rate of the DMT system is also maximized. This idea was originally presented in [18] as a "per-tone equalization" (PTEQ) architecture. An alternative formulation, called the "Time-Domain Equalizer Filter Bank" (TEQFB), is given in [17].

In terms of the common formulation given by (1), a single generalized Rayleigh quotient $(M=1)$ must be individually maximized for each subchannel. This procedure is repeated 
for all $N_{u}$ data-carrying subchannels. Because each Rayleigh quotient is individually maximized, one can guarantee that an optimum solution can be found (in contrast to the previous section). Although the problem has been simplified into a form that is easier to solve, the total complexity will still be high since we have to perform many simple optimizations rather than a single complicated optimization.

\section{A. Per-tone equalization}

The PTEQ [18] is based on the idea that the TEQ and the demodulating DFT can be interchanged. The equalizer is implemented after the DFT, hence it can be considered as "frequency domain" equalization. This allows for a decoupling of the equalizer design per tone $i$, with the advantage that the PTEQ with $L_{w}+1$ taps per tone performs as well as and usually better (in terms of bit rate) than a single TEQ with $L_{w}+1$ taps, with comparable complexity during data transmission. The idea behind the PTEQ can be summarized by noting that for a TEQ, the equalized $i$-th DFT output $U_{i}^{k}$ (tone $i$, symbol $k$ ) can be obtained in two ways:

$$
U_{i}^{k}=\mathbf{f}_{i}\left(\mathbf{M}^{k} \mathbf{w}\right)=\left(\mathbf{f}_{i} \mathbf{M}^{k}\right) \mathbf{w} .
$$

Here, $\mathbf{M}^{k}$ is an $N \times\left(L_{w}+1\right)$ Toeplitz matrix of received samples $\mathbf{y}^{k}[l]$ of the current symbol $k$ as in (10), and $\mathbf{f}_{i}$ is the $i$-th row of the DFT matrix. The left-hand side of (34) represents the classical convolution of the received signal $\mathbf{y}^{k}=\left[y\left(s k+\nu-L_{w}+1\right), \cdots, y(s(k+1))\right]^{T}$ with the TEQ, $\mathbf{M}^{k} \mathbf{w}$, followed by the DFT (Fig. 1). The right-hand side of (34) implies that the equalized $i$-th DFT output $U_{i}^{k}$ can also be seen as a linear combination by $\mathbf{w}$ of $L_{w}+1$ consecutive outputs of a sliding FFT on the $i$-th tone, applied to the unequalized received signal $\mathbf{y}^{k}[18]$.

A symbol estimate $\hat{X}_{i}^{k}$ is then obtained as

$$
\hat{X}_{i}^{k}=\left(\mathbf{f}_{i} \mathbf{M}^{k}\right) \underbrace{\mathbf{w} D_{i}}_{\mathbf{w}_{i}},
$$

where now a tone-dependent and complex set of coefficients $\mathbf{w}_{i}$ has been introduced by combining the TEQ $\mathbf{w}$ and the FEQ $D_{i}$. To avoid the need for $L_{w}+1$ consecutive FFT operations per symbol $\mathbf{f}_{i} \mathbf{M}^{k}$ in (35), the Toeplitz structure of $\mathbf{M}^{k}$ can be exploited:

$$
\begin{aligned}
& \mathbf{f}_{i} \mathbf{M}^{k}[:, l+1]=\alpha^{i-1} \mathbf{f}_{i} \mathbf{M}^{k}[:, l]+(36) \\
& \underbrace{\left(\mathbf{y}^{k}\left[L_{w}-l\right]-\mathbf{y}^{k}\left[L_{w}-l+N\right]\right)}_{\Delta \mathbf{y}^{k}[l]}, \quad l=1, \cdots, L_{w} .
\end{aligned}
$$

Here, $\alpha=\exp (-j 2 \pi / N)$ and $\mathbf{M}^{k}[:, l]$ denotes the $l$-th column of $\mathbf{M}^{k}$. In other words, the DFT of a column of $\mathbf{M}^{k}$ can be derived from the DFT of its previous column plus some correction term. An efficient implementation of (35) then only needs a single FFT per symbol. The symbol estimate $\hat{X}_{i}^{k}$ is obtained by linearly combining the unequalized $i$-th DFT output $Y_{i}^{k}$ with $L_{w}$ real difference terms $\Delta \mathbf{y}^{k}[l], l=$ $1, \cdots, L_{w}$, as defined in (37):

$$
\hat{X}_{i}^{k}=\underbrace{\left[Y_{i}^{k}, \quad \boldsymbol{\Delta} \mathbf{y}^{k}[1], \quad \cdots, \quad \boldsymbol{\Delta} \mathbf{y}^{k}\left[L_{w}\right]\right]}_{\mathbf{Z}_{i}^{k}} \mathbf{v}_{i} .
$$

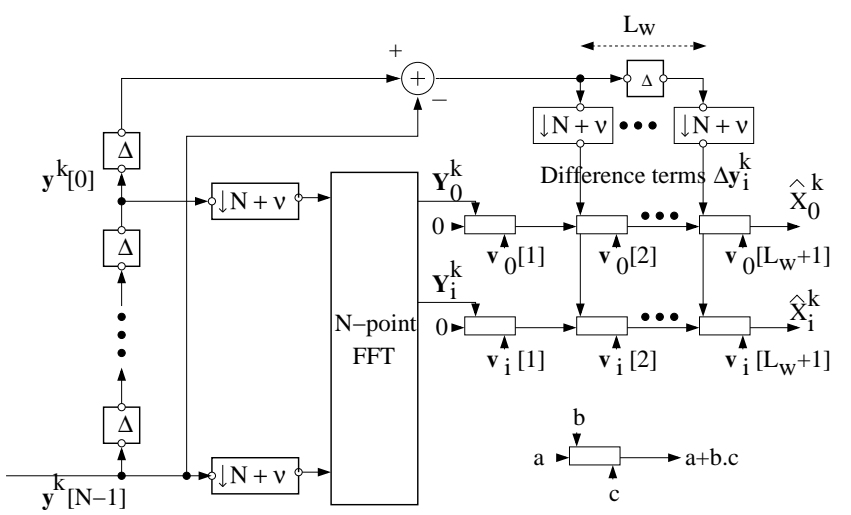

Fig. 2. PTEQ architecture: Channel Equalization Block at the Receiver

Here, $\mathbf{v}_{i}$ are the complex PTEQ coefficients, related to $\mathbf{w}_{i}$ in (35), by

$$
\begin{aligned}
\mathbf{v}_{i}[l] & =\alpha^{i-1} \mathbf{v}_{i}[l+1]+\mathbf{w}_{i}[l], \quad l=1, \cdots, L_{w} \\
\mathbf{v}_{i}\left[L_{w}+1\right] & =\mathbf{w}_{i}\left[L_{w}+1\right] .
\end{aligned}
$$

Fig. 2 depicts the PTEQ scheme. An alternate derivation based on an infinite-impulse response (IIR) channel model in [46] led to a generalized PTEQ which exploits pilot and unused tones.

To determine a bit rate maximizing set of PTEQ coefficients $\mathbf{v}_{i}$ for each subchannel, it suffices to solve a linear MMSE cost function for each tone:

$$
\min _{\mathbf{v}_{i}} J\left(\mathbf{v}_{i}\right)=\min _{\mathbf{v}_{i}} \mathcal{E}\left\{\left|\mathbf{Z}_{i}^{k} \mathbf{v}_{i}-X_{i}^{k}\right|^{2}\right\}
$$

There are several strategies for solving (41):

- solving a least squares problem per tone as in [18], based on channel and noise estimates;

- an efficient blind or training-based adaptive algorithm [47], [48];

- the classical MMSE solution, given by

$$
\mathbf{v}_{i}=\mathcal{E}\left\{\left(\mathbf{Z}_{i}^{k}\right)^{H} \mathbf{Z}_{i}^{k}\right\}^{-1} \mathcal{E}\left\{\left(\mathbf{Z}_{i}^{k}\right)^{H}\left(X_{i}^{k}\right)\right\}
$$

- a generalized eigenvalue problem (3) could be solved for each tone $i$ with [49]

$$
\begin{aligned}
& \mathbf{A}_{i}=\mathcal{E}\left\{\left(\mathbf{Z}_{i}^{k}\right)^{H} \mathbf{Z}_{i}^{k}\right\} \\
& \mathbf{B}_{i}=\mathcal{E}\left\{\left(\mathbf{Z}_{i}^{k}\right)^{H}\left(X_{i}^{k}\right)\right\} \mathcal{E}\left\{\mathbf{Z}_{i}^{k}\left(X_{i}^{k}\right)^{*}\right\},
\end{aligned}
$$

which is equivalent to the MMSE solution.

\section{B. Time domain equalizer filter bank}

An alternative scheme with an equalizer $\mathbf{w}_{i}$ for each subchannel $i$ is the TEQ Filter Bank (TEQFB) [17], as depicted in Fig. 3. Each TEQ $\mathbf{w}_{i}$ filters the received signal $\mathbf{y}^{k}$. All $N \times 1$ TEQ output vectors $\mathbf{u}_{i}^{k}=\left[u_{i}(k s+\nu+1), \cdots, u_{i}((k+1) s)\right]$ are fed into a Goertzel filter bank [50]. Each Goertzel filter $\mathbf{f}_{i}$ is tuned to the frequency of subchannel $i$ and computes a single point DFT: $\mathbf{f}_{i} \mathbf{u}_{i}^{k}=\mathbf{f}_{i}\left(\mathbf{M}^{k} \mathbf{w}_{i}\right)$ (with $\mathbf{M}^{k}$ a Toeplitz matrix of received samples as in (10)). Finally, a 1-tap FEQ $D_{i}$ is applied to each output to give a symbol estimate $\hat{X}_{i}^{k}$. 


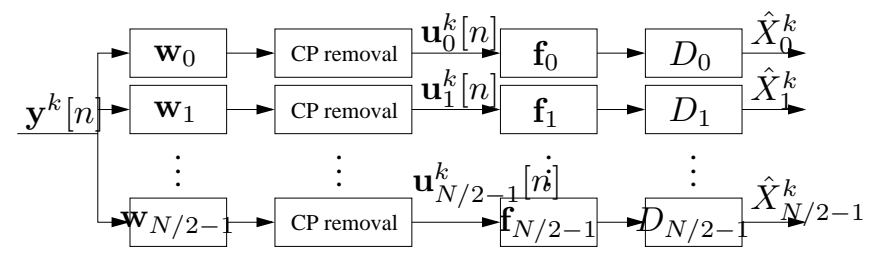

Fig. 3. TEQFB architecture. $\hat{X}_{i}^{k}$ is the estimate of $X_{i}^{k}$, the transmitted data on tone $i$ for symbol $k$.

The subchannel SNR model for the TEQFB is identical to the model for the MDR TEQ presented in Section III-C. The difference is that now we maximize each subchannel SNR by its own TEQ, rather than using a single TEQ to maximize the bit allocation as a function of all of the subchannel SNRs. The dependence of the number of bits per symbol on the TEQ is established using (7) and (8).

\section{PTEQ or TEQFB?}

The TEQFB in [17] is based on an approximate SNR model (7) based on channel and noise models, whereas the PTEQ in [18] optimizes the true subchannel SNRs. Provided that the same a priori knowledge about channel and noise is used, an exact SNR model for the TEQFB is applied, and complexvalued TEQs are allowed, then the TEQFB and the PTEQ give the same performance, which is an upper bound for the singleTEQ-based receiver. Due to its large computational burden, the TEQFB in [17] is not proposed as a practical approach, but as a bound to the performance that can be achieved with a single FIR TEQ. On the other hand, the PTEQ has roughly the same computational complexity as a TEQ-based receiver during data transmission, though the PTEQ and TEQFB both have high training complexity.

\section{Single QuOTIEnT CASES}

The vast majority of TEQ designs can be formulated as the maximization of a generalized Rayleigh quotient, as in (2). This section reviews the literature, advantages, and disadvantages of these designs. Historically, most of these designs were proposed before the designs in Sections III and IV that attempt to maximize bit rate. However, since bit rate maximization is the ultimate goal, one can view the single quotient designs in this section as approximations of the multiple-quotient, bit rate maximizing designs.

\section{A. Minimum mean square error (MMSE)}

In the seventies, an MMSE method was devised to shorten the channel impulse response for maximum likelihood sequence estimation (MLSE) [29]. The objective was to design a filter prior to the Viterbi algorithm, which is frequently used for MLSE. This pre-filtering attempts to reduce the channel memory, resulting in an exponential decrease in computational complexity of the Viterbi algorithm.

In the early nineties, Chow and Cioffi [51] extended the MMSE channel shortening problem to time domain equalization in multicarrier systems. In [51], a finite and an infinite length TEQ are computed to shorten the channel impulse

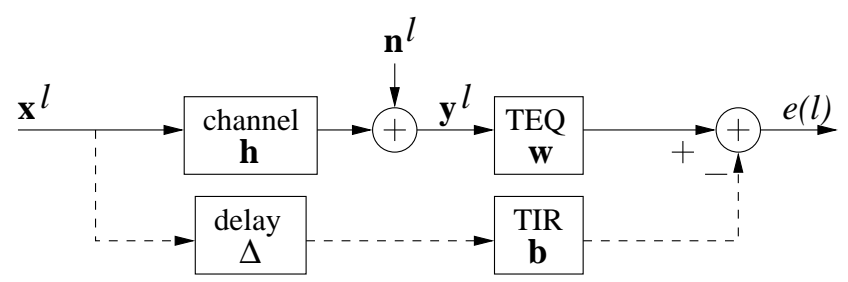

Fig. 4. Block diagram used for MMSE channel shortening.

response (CIR) to a $\nu+1$ tap target impulse response (TIR). In this paper, we will focus on the finite length case.

The MMSE TEQ design is depicted in Fig. 4. Note that it operates independently of the DMT block structure, hence here we use the sample index $l$ and not the block index $k$. Define $\mathbf{x}^{l}=[x(l), \cdots, x(l-\nu)]^{T}$ and $\mathbf{y}^{l}=\left[y(l), \cdots, y\left(l-L_{w}\right)\right]^{T}$. The transmitted sequence $x(l)$ is passed through the CIR $\mathbf{h}$ and is equalized by the TEQ $\mathbf{w}$. The equalizer output is compared with the input signal filtered by the TIR $\mathbf{b}$ and delayed with $\Delta$. The difference sequence $e(l)$ is then minimized in the mean square sense with respect to $\mathbf{w}$ and $\mathbf{b}$, i.e. the cost function can be expressed as

$$
\begin{aligned}
J(\mathbf{w}, \mathbf{b}) & =\mathcal{E}\left\{e^{2}(l)\right\}=\mathcal{E}\left\{\left(\mathbf{w}^{T} \mathbf{y}^{l}-\mathbf{b}^{T} \mathbf{x}^{l-\Delta}\right)^{2}\right\}, \\
& =\mathbf{w}^{T} \mathbf{R}_{y} \mathbf{w}+\mathbf{b}^{T} \mathbf{R}_{x} \mathbf{b}-2 \mathbf{b}^{T} \mathbf{R}_{y x}^{T}(\Delta) \mathbf{w},
\end{aligned}
$$

where $\mathbf{R}_{x}=\mathcal{E}\left\{\mathbf{x}^{l}\left(\mathbf{x}^{l}\right)^{T}\right\}, \mathbf{R}_{y}=\mathcal{E}\left\{\mathbf{y}^{l}\left(\mathbf{y}^{l}\right)^{T}\right\}$, and $\mathbf{R}_{y x}(\Delta)=$ $\mathcal{E}\left\{\mathbf{y}^{l}\left(\mathbf{x}^{l-\Delta}\right)^{T}\right\}$, which is a function of the delay parameter $\Delta$. For a given $\mathbf{w}$, there will be an optimal setting for $\mathbf{b}$, and vice versa. Hence, either can be treated as a function of the other. This functional relation of the optimal vectors can be found via

$$
\begin{aligned}
& \nabla_{\mathbf{w}} J=0 \quad \rightarrow \quad \mathbf{w}=\mathbf{R}_{y}^{-1} \mathbf{R}_{y x} \mathbf{b} \\
& \nabla_{\mathbf{b}} J=0 \quad \rightarrow \quad \mathbf{b}=\mathbf{R}_{x}^{-1} \mathbf{R}_{x y} \mathbf{w}
\end{aligned}
$$

which allows reformulation of (46) as a function of $\mathbf{w}$ or $\mathbf{b}$ alone [29].

The trivial all-zero solution can be avoided by adding a constraint on the TEQ or TIR [8], [51], [52], [53], [54], [55]. The MMSE optimization problem with various constraints can be cast into the general problem formulation of (2) with different $\mathbf{A}$ and $\mathbf{B}$ matrices for the different constraints, such as:

1) unit-norm constraint on the TEQ [54], [55], i.e. $\mathbf{w}^{T} \mathbf{w}=$ 1. To see how to put this in the form of (2), substitute (48) into (46):

$$
\begin{aligned}
J(\mathbf{w})= & \mathbf{w}^{T} \mathbf{R}_{y} \mathbf{w}+\left(\mathbf{w}^{T} \mathbf{R}_{y x} \mathbf{R}_{x}^{-1}\right) \mathbf{R}_{x}\left(\mathbf{R}_{x}^{-1} \mathbf{R}_{x y} \mathbf{w}\right) \\
-2\left(\mathbf{w}^{T} \mathbf{R}_{y x} \mathbf{R}_{x}^{-1}\right) \mathbf{R}_{x y} \mathbf{w} & \underbrace{\left(\mathbf{R}_{y}-\mathbf{R}_{y x} \mathbf{R}_{x}^{-1} \mathbf{R}_{x y}\right)}_{\mathbf{A}} \mathbf{w} .
\end{aligned}
$$

Minimizing $J(\mathbf{w})$ while maintaining $\mathbf{w}^{T}\left(\mathbf{I}_{L_{w}+1}\right) \mathbf{w}=1$ requires solving (2) with

$$
\begin{aligned}
& \mathbf{A}=\mathbf{R}_{y}-\mathbf{R}_{y x} \mathbf{R}_{x}^{-1} \mathbf{R}_{x y}, \\
& \mathbf{B}=\mathbf{I}_{L_{w}+1} .
\end{aligned}
$$

As discussed in Section II, the optimal TEQ is the eigenvector corresponding to the smallest eigenvalue of 
A. In [16], [54], [55], the unsatisfactory performance of this constraint was reported. Under this constraint the TEQ typically boosts 'out of band' noise, which leaks into the band of interest due to the poor spectral containment of the demodulating DFT [16]. In order to concentrate the TEQ energy into the desired passband region, virtual (i.e. mathematical) noise can be injected into the stopband, using a modified $\mathbf{A}$ matrix:

$$
\mathbf{A}=\left(\mathbf{R}_{y}+\mu \mathbf{D}\right)-\mathbf{R}_{y x} \mathbf{R}_{x}^{-1} \mathbf{R}_{x y} .
$$

Here, the scalar $\mu$ controls the virtual noise level, and $\mathbf{D}$ is as in (22). The TEQ tries to suppress the virtual noise, thereby lowering the undesired noise enhancement [16].

2) unit-energy constraints [54], i.e. $\mathbf{w}^{T} \mathbf{R}_{y} \mathbf{w}=1$, or $\mathbf{b}^{T} \mathbf{R}_{x} \mathbf{b}=1$, or $\mathbf{w}^{T} \mathbf{R}_{y} \mathbf{w}=1 \& \mathbf{b}^{T} \mathbf{R}_{x} \mathbf{b}=1$ :

$$
\begin{aligned}
& \mathbf{A}=\mathbf{R}_{y}-\mathbf{R}_{y x} \mathbf{R}_{x}^{-1} \mathbf{R}_{x y}, \\
& \mathbf{B}=\mathbf{R}_{y} .
\end{aligned}
$$

These different unit-energy constraints remarkably lead to the same TEQ coefficients, up to a scaling factor, which can be incorporated into the one-tap FEQs [54].

3) unit-norm constraint on the TIR [52], i.e. $\mathbf{b}^{T} \mathbf{b}=1$. If $\mathbf{x}^{k}$ is white, then $\mathbf{R}_{x}$ is identity, so $\mathbf{b}^{T} \mathbf{R}_{x} \mathbf{b}=\mathbf{b}^{T} \mathbf{b}$ and the previous case with $\mathbf{R}_{x}=\mathbf{I}_{\nu+1}$ yields the optimal TEQ. Whether $\mathbf{x}^{k}$ is white or not, (2) can be reformulated with $\widehat{\mathbf{w}}=\mathbf{b}$ and [29]

$$
\begin{aligned}
& \mathbf{A}=\mathbf{R}_{x}-\mathbf{R}_{x y} \mathbf{R}_{y}^{-1} \mathbf{R}_{y x}, \\
& \mathbf{B}=\mathbf{I}_{\nu+1} .
\end{aligned}
$$

After calculating the solution for $\mathbf{b}$, the TEQ coefficients can be obtained using (47).

4) unit-tap constraint on the TIR [52], i.e. $\mathbf{e}_{j}^{T} \mathbf{b}= \pm 1$, where $\mathbf{e}_{j}$ is the elementary vector with element one in the $j$ th position. The optimal TIR uses (2) with $\widehat{\mathbf{w}}=\mathbf{b}$ and

$$
\begin{aligned}
\mathbf{A} & =\mathbf{R}_{x}-\mathbf{R}_{x y} \mathbf{R}_{y}^{-1} \mathbf{R}_{y x}, \\
\mathbf{B} & =\mathbf{e}_{j} \mathbf{e}_{j}^{T} .
\end{aligned}
$$

After calculating the solution for $\mathbf{b}$, the TEQ coefficients can be obtained using (47).

5) unit-tap constraint on the TEQ, i.e. $\mathbf{e}_{j}^{T} \mathbf{w}= \pm 1$ :

$$
\begin{aligned}
& \mathbf{A}=\mathbf{R}_{y}-\mathbf{R}_{y x} \mathbf{R}_{x}^{-1} \mathbf{R}_{x y}, \\
& \mathbf{B}=\mathbf{e}_{j} \mathbf{e}_{j}^{T} .
\end{aligned}
$$

The first TEQ algorithm proposed for DMT transmission [56] also falls into this category. During modem initialization, an IIR channel model (with $\nu$ zeros and $L_{w}$ poles) is derived,

$$
H(z)=\frac{B(z)}{A(z)}=\frac{z^{-\Delta} \sum_{l=0}^{\nu} b_{l} z^{-l}}{1+\sum_{l=1}^{L_{w}} a_{l} z^{-l}}
$$

where $z$ is the $\mathcal{Z}$-transform variable. Based on this model, the receiver then sets its TEQ taps to $a_{l}$, for $l=$

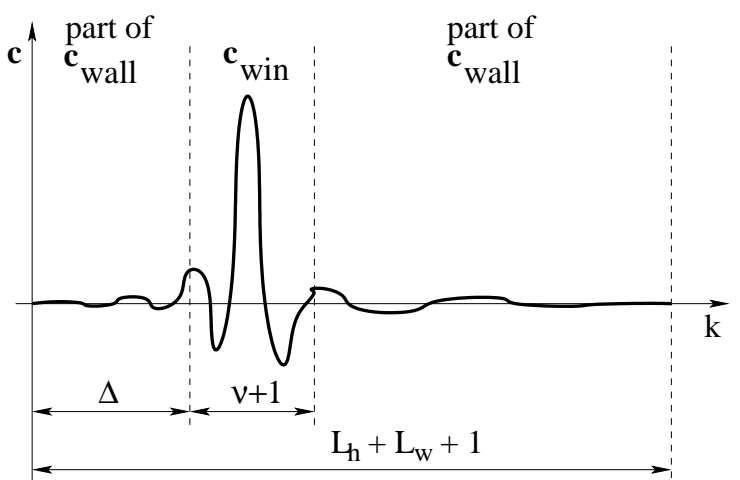

Fig. 5. The "window" and "wall" parts of the effective channel.

$0, \ldots, L_{w}$, such that the effective channel corresponds to the numerator of $H(z)$, which is confined in extent to be $\mathrm{CP}$ length plus one taps. One can easily verify that a classical linear prediction method to estimate the numerator and denominator coefficients of $H(z)$ is equivalent to computing an MMSE TEQ with $\mathbf{e}_{1}^{T} \mathbf{w}=1$. This idea was extended to a multiple-input-single-output filter bank at the receiver in [57].

MMSE TEQ design has been extensively studied. Chow and Cioffi's basic results of [51] were further explored in [52] with emphasis on unit-tap and unit-norm constraints on the TIR. The authors of [52] have shown that a unit-norm constrained TIR results in a lower MMSE than a unit-tap constrained TIR, and concluded that the unit-norm constraint results in better performance. However, the MSE is not directly related to the bit rate [35], [43] hence it is difficult to predict which constraint is preferable. In an attempt to improve the performance of the MMSE design, Van Acker et al. modified the cost function with frequency weighting to disregard unused frequency bins [55], [58], [59]. Although the authors report some improvement in bit rate, this approach still does not maximize the bit rate. Moreover, the bit rate is a non-smooth function of $\Delta$, and thus optimizing the delay requires a global search [18].

\section{B. Maximum shortening SNR (MSSNR)}

In 1996, Melsa, Younce, and Rohrs proposed the maximum shortening signal-to-noise ratio (MSSNR) method [31], which is based solely on shortening the CIR. The MSSNR technique [31] attempts to minimize the energy outside a length $\nu+1$ window of the effective channel $\mathbf{c}=\mathbf{h} \star \mathbf{w}$ (called the 'wall'), while constraining the energy in the desired window of $\mathbf{c}$ to one, as shown in Fig. 5. Define

$$
\mathbf{H}_{w i n}=\left[\begin{array}{ccc}
h_{\Delta} & \ldots & h_{\Delta-L_{w}} \\
\vdots & \ddots & \vdots \\
h_{\Delta+\nu} & \ldots & h_{\Delta+\nu-L_{w}}
\end{array}\right]
$$

$\mathbf{H}_{\text {wall }}=$

$$
\left[\begin{array}{cccc|cccc}
h_{0} & h_{1} & \ldots & h_{\Delta-1} & h_{\Delta+\nu+1} & \ldots & 0 & 0 \\
0 & h_{0} & \ddots & \vdots & \vdots & \ddots & \ddots & \ddots \\
\ddots & \ddots & \ddots & h_{\Delta-L_{w}-1} & h_{\Delta+\nu-L_{w}+1} & \ldots & h_{L_{h}-1} & h_{L_{h}}
\end{array}\right]^{T}
$$


The window and wall portions of $\mathbf{c}$, as depicted in Fig. 5, are denoted $\mathbf{c}_{\text {win }}$ and $\mathbf{c}_{\text {wall }}$, respectively. The expressions for the energy outside and inside the window can be written as

$$
\begin{aligned}
\mathbf{c}_{\text {wall }}^{T} \mathbf{c}_{\text {wall }} & =\mathbf{w}^{T} \mathbf{H}_{\text {wall }}^{T} \mathbf{H}_{\text {wall }} \mathbf{w}, \\
\mathbf{c}_{\text {win }}^{T} \mathbf{c}_{\text {win }} & =\mathbf{w}^{T} \mathbf{H}_{\text {win }}^{T} \mathbf{H}_{\text {win }} \mathbf{w},
\end{aligned}
$$

respectively. The final TEQ coefficients are found as

$$
\min _{\mathbf{w}} \mathbf{w}^{T} \mathbf{H}_{\text {wall }}^{T} \mathbf{H}_{\text {wall }} \mathbf{w} \quad \text { s.t. } \quad \mathbf{w}^{T} \mathbf{H}_{\text {win }}^{T} \mathbf{H}_{\text {win }} \mathbf{w}=1 \text {. (67) }
$$

When $\mathbf{H}_{w i n}^{T} \mathbf{H}_{w i n}$ has a non-empty null space, i.e. when $\mathbf{H}_{w i n}$ has more columns than rows $\left(L_{w}>\nu\right), \mathbf{H}_{w i n}^{T} \mathbf{H}_{w i n}$ becomes non-invertible and solving (67) is rather complicated [31].

Alternatively, one can maximize the windowed energy, while constraining the wall energy, as suggested in [60] and later in [61]. Since $\mathbf{H}_{\text {wall }}^{T} \mathbf{H}_{\text {wall }}$ is always positive definite when $L_{h} \geq \nu+1$, the latter approach is preferred and reduces to solving (2) with

$$
\begin{aligned}
& \mathbf{A}=\mathbf{H}_{w a l l}^{T} \mathbf{H}_{w a l l} \\
& \mathbf{B}=\mathbf{H}_{w i n}^{T} \mathbf{H}_{w i n}
\end{aligned}
$$

Maximizing window to wall energy provides the same TEQ as maximizing overall channel energy to wall energy [62], [63].

The MSSNR approach tacitly assumes that the input signal is white. In the absence of noise, for a white input signal the MSSNR approach is equivalent to the MMSE design [64]. The MSSNR TEQ ignores noise, so it may be referred to as a zeroforcing (ZF) design. The MSSNR method can be extended to the noisy case by adding a noise correlation matrix to $\mathbf{A}$, i.e. [65]

$$
\begin{aligned}
& \mathbf{A}=\mathbf{H}_{\text {wall }}^{T} \mathbf{H}_{\text {wall }}+\mathbf{R}_{n}, \\
& \mathbf{B}=\mathbf{H}_{\text {win }}^{T} \mathbf{H}_{\text {win }} .
\end{aligned}
$$

The infinite length MSSNR TEQ is always symmetric or skew-symmetric [30], [65], [66]. Interestingly, the finite length MSSNR TEQ is almost always nearly symmetric. Thus, design complexity can be dramatically reduced by forcing a perfectly symmetric TEQ [30], [65] by rewriting $\mathbf{w}^{T} \mathbf{A w}$ (with $\mathbf{A}$ as in (68) or (70)) as

$$
\begin{aligned}
& \underbrace{\left[\begin{array}{ll}
\widetilde{\mathbf{w}}^{T}, & \widetilde{\mathbf{w}}^{T} \mathbf{J}
\end{array}\right]}_{\mathbf{w}^{T}} \underbrace{\left[\begin{array}{cc}
\mathbf{A}_{11}, & \mathbf{A}_{12} \\
\mathbf{A}_{21}, & \mathbf{A}_{22}
\end{array}\right]}_{\text {MSSNR } \mathbf{A}} \underbrace{\left[\begin{array}{c}
\widetilde{\mathbf{w}} \\
\mathbf{J} \widetilde{\mathbf{w}}
\end{array}\right]}_{\mathbf{w}} \\
& =\widetilde{\mathbf{w}}^{T} \underbrace{\left[\mathbf{A}_{11}+\mathbf{J} \mathbf{A}_{21}+\mathbf{A}_{12} \mathbf{J}+\mathbf{J} \mathbf{A}_{22} \mathbf{J}\right]}_{\text {Sym-MSSNR A }} \widetilde{\mathbf{w}},
\end{aligned}
$$

where $\mathbf{J}$ is the square matrix with ones on the anti-diagonal and $\widetilde{\mathbf{w}}$ is half the size of $\mathbf{w}$. A similar redefinition holds for $\mathbf{B}$. The desired symmetric TEQ is obtained via (2) with $\widehat{\mathbf{w}}=\widetilde{\mathbf{w}}$ and the $\mathbf{A}$ and $\mathbf{B}$ matrices redefined as

$$
\begin{aligned}
& \mathbf{A}=\mathbf{A}_{11}+\mathbf{J} \mathbf{A}_{21}+\mathbf{A}_{12} \mathbf{J}+\mathbf{J} \mathbf{A}_{22} \mathbf{J} \\
& \mathbf{B}=\mathbf{B}_{11}+\mathbf{J B}_{21}+\mathbf{B}_{12} \mathbf{J}+\mathbf{J B}_{22} \mathbf{J}
\end{aligned}
$$

In [65], [67], it was reported that symmetric MSSNR TEQs have a comparable performance with respect to the MSSNR design of [31], with reduced computational complexity.
C. Multicarrier equalization by restoration of redundancy (MERRY)

In [33], one of the few blind channel-shortening algorithms was presented. This method, called MERRY, exploits the CP redundancy to force the last sample in the equalized $\mathrm{CP}$ to be equal to the last sample in the equalized symbol. The cost function that reflects this principle is

$$
J(\mathbf{w})=\mathcal{E}\left\{|u(s k+\nu+\Delta)+u(s k+\nu+N+\Delta)|^{2}\right\},
$$

where $u(l)$ denotes the signal after the TEQ at the $l$-th time index. From (76), it follows that MERRY attempts to produce a windowed effective channel of $\nu$ taps instead of $\nu+1$ [33]. If the input signal is white, minimizing (76) also minimizes the "wall" of the effective channel (like the MSSNR design) under the constraint $\mathbf{w}^{T} \mathbf{w}=1$ (or a unit energy constraint [63]) while limiting the noise gain [33]. The MERRY design can be formulated as a single generalized Rayleigh quotient optimization as in (2) with

$$
\begin{aligned}
& \mathbf{A}=\tilde{\mathbf{H}}_{\text {wall }}^{T} \tilde{\mathbf{H}}_{\text {wall }}+\mathbf{R}_{n}, \\
& \mathbf{B}=\mathbf{I}_{L_{w}+1},
\end{aligned}
$$

where $\tilde{\mathbf{H}}_{\text {wall }}$ contains one extra row compared to the MSSNR matrix (64).

\section{Minimum intersymbol interference (Min-ISI)}

A generalization of the MSSNR method was given in [35] and [68], referred to as the minimum ISI (min-ISI) method. The Min-ISI design can also be thought of as an approximation of the MBR method of Section III-D.

Arslan, Evans, and Kiaei [35] model the sub-channel SNR as was done for the MBR method in Section III-D. However, according to [68], the matched filter bound on the SNR is obtained when each sub-carrier ISI term of (20) is forced to zero. As a consequence, they propose to minimize a weighted sum of the sub-channel ISI terms. The resulting TEQ design is of the form of (2) with

$$
\begin{aligned}
\mathbf{A} & =\mathbf{H}^{T} \mathbf{D}^{T}\left(\sum_{i \in \mathcal{S}} \mathbf{f}_{i}^{H} S_{x, i} \mathbf{f}_{i}\right) \mathbf{D H}, \\
\mathbf{B} & =\mathbf{H}_{w i n}^{T} \mathbf{H}_{w i n},
\end{aligned}
$$

where $\mathbf{D}$ is as in (22), $\mathcal{S}$ denotes the set of used tones, and the constraint $\mathbf{w}^{T} \mathbf{B w}=1$ prevents the trivial all-zero TEQ solution. In [35], channel noise coloration was taken into account by modifying (79) and (80) into

$$
\begin{aligned}
& \mathbf{A}=\mathbf{H}^{T} \mathbf{D}^{T}\left(\sum_{i \in \mathcal{S}} \mathbf{f}_{i}^{H} \frac{S_{x, i}}{S_{n, i}} \mathbf{f}_{i}\right) \mathbf{D H} \\
& \mathbf{B}=\mathbf{H}_{\text {win }}^{T} \mathbf{H}_{w i n} .
\end{aligned}
$$

The conventional subchannel SNR ratio of $\frac{S_{x, i}}{S_{n, i}}$ in (81) forces the ISI to be placed in subchannels with low conventional subchannel SNR. Note that DH is a zero-padded version of $\mathbf{H}_{\text {wall }}$. Comparing (68) and (69) to (81) and (82), the residual ISI is now shaped in the frequency domain. The minISI method [35] is a generalization of the MSSNR method 
[31], since both methods would be equivalent if the SNR were constant over all sub-channels and if all sub-channels were used. An improved Min-ISI method [69] generalizes ISI shaping function in frequency domain and further reduces implementation cost.

The dual-path TEQ [70] makes use of the Min-ISI design. One TEQ is designed for all of the tones, and then a second TEQ is designed in parallel using the Min-ISI method for a small subset of tones. The subset is chosen as the lowfrequency tones which are expected to have a high bit rate.

The min-ISI method resulted from applying a simplification to the MBR method to make the approach tractable. As such, it is suboptimal in terms of bit rate performance. As the demodulating DFT length is finite, sub-carriers are not perfectly orthogonal, which results in inter-carrier interference (ICI). The ICI-noise component is neglected in (20). In addition, the signal path gain of (21) is an approximation. In practice, the head and the tail of the effective channel will contribute to the useful signal component [15].

\section{E. Minimum delay spread (MDS)}

The taps of $\mathbf{c}$ exceeding the CP length cause ISI and ICI, but the interference levels depend on the taps' distances to the prefix and their energy [71]. Therefore, Schur and Speidel [71] propose to minimize the square of the delay spread of $\mathbf{c}$, where the delay spread is given by

$$
D=\sqrt{\frac{1}{E} \sum_{n=0}^{L_{c}}(n-\bar{n})^{2}|\mathbf{c}[n]|^{2}} .
$$

Here, $E=\mathbf{c}^{T} \mathbf{c}=\mathbf{w}^{T} \mathbf{H}^{T} \mathbf{H w}$, and $\bar{n}$ is a user-defined "center of mass." This results in (2) with

$$
\begin{aligned}
\mathbf{A} & =\mathbf{H}^{T} \mathbf{Q H}, \\
\mathbf{B} & =\mathbf{H}^{T} \mathbf{H},
\end{aligned}
$$

where $\mathbf{Q}=\operatorname{diag}\left\{\left[(0-\bar{n})^{2}, \quad(1-\bar{n})^{2}, \quad \ldots, \quad\left(L_{w}+L_{h}-\right.\right.\right.$ $\left.\left.\bar{n})^{2}\right]\right\}$ is a diagonal weighting matrix. The minimum interblock interference (Min-IBI) method [72] is a similar MSSNR variant that weights the ISI terms linearly with their distance from the channel window.

Since the MDS TEQ does not exploit the cyclic prefix redundancy, it attempts to shorten the effective channel to a single spike. Since MDS TEQ design is quite similar to MSSNR TEQ design, except for a quadratic instead of a wall penalty function [73], the advantages and drawbacks mentioned in Section V-B also apply here.

\section{F. Carrier nulling algorithm (CNA)}

In a typical DMT/OFDM system, some frequency bins transmit only zeros, the so-called null-carriers. In [74], the authors propose a blind method to combat channel dispersion based on the minimization of the average DFT-output energy of the null carriers. The TEQ can be designed to force the received symbols on the null-carriers to zero by minimizing the cost function

$$
\begin{aligned}
J & =\sum_{i \in \overline{\mathcal{S}}} \mathcal{E}\left\{\left|U_{i}^{k}\right|^{2}\right\}, \\
& =\mathbf{w}^{T}\left(\mathbf{P}_{\text {cna }}+\mathbf{Q}_{\text {cna }}\right) \mathbf{w},
\end{aligned}
$$

where $\overline{\mathcal{S}}$ represents the set of null-carriers, $U_{i}^{k}$ is the DFT output on tone $i$, and $\mathbf{P}_{\text {cna }}$ and $\mathbf{Q}_{\text {cna }}$ denote signal and noise dependent matrices respectively (see [74] for complete definitions). The constraint $\mathbf{w}^{T} \mathbf{w}=1$, is used to avoid the all-zero solution. The CNA TEQ then solves (2) with

$$
\begin{aligned}
& \mathbf{A}=\mathbf{P}_{\text {cna }}+\mathbf{Q}_{\text {cna }}, \\
& \mathbf{B}=\mathbf{I}_{L_{w}+1} .
\end{aligned}
$$

Although [74] presented a low-complexity, blind, adaptive minimization procedure for (86), the CNA criterion only considers unused carriers without regard to the carriers of interest. This will not necessarily lead to channel shortening, nor equalization of the useful carriers. Specifically, de Courville et al. claim that CNA leads to shortening to a single spike [74] rather than to a window, though Romano and Barbarossa state that an MSSNR solution can be achieved by frequencyhopping the null tones [75].

\section{EXCEPTIONS TO THE COMMON FORMULATION}

This section addresses two designs that do not fit into the framework of Section II. Henkel and Kessler [76] presented one of the first attempts to improve upon the MGSNR design of [43]. Their subchannel SNR model includes the leakage effect of the DFT on the noise as well as ISI. The leakage effect comes from the implicit rectangular time-domain window of the DFT. ICI is neglected and all carriers are assumed to be active. Based on this model, any multidimensional optimization algorithm can be used to optimize the bitrate. Their subchannel SNR model renders the method outside of our general framework (1).

The sum-squared auto-correlation minimization (SAM) algorithm [77], meant for blind, adaptive channel shortening, shortens auto-correlation of the effective channel $\mathbf{c}=\mathbf{h} \star \mathbf{w}$ :

$$
\min _{\mathbf{w}} J(\mathbf{w})=\sum_{l=\nu+1}^{L_{c}}\left|R_{\mathbf{c}}[l]\right|^{2} \quad \text { subject to }\|\mathbf{w}\|^{2}=1
$$

where $R_{\mathbf{c}}[l]=\sum_{n=0}^{L_{c}} \mathbf{c}[n] \mathbf{c}[n-l]$ is the autocorrelation sequence of the effective channel $\mathbf{c}$. The constraint $\|\mathbf{w}\|^{2}=1$ prevents the all-zero solution. If the transmit sequence is white and wide-sense stationary, the cost function can be written as a function of the TEQ output sequence $u(n)$,

$$
J(\mathbf{w})=\sum_{l=\nu+1}^{L_{c}}|\mathcal{E}\{u(n) u(n-l)\}|^{2} .
$$

As it is fourth-order in $\mathbf{w}$ (and hence multimodal), proper initialization is required.

\section{COMMUNICATIONS PERFORMANCE EVALUATION}

This section presents a performance comparison of the various designs discussed in the previous sections. Section VII-A describes the synthetic data and results, Section VII-B reports the performance for measured DSL channels, and Section VII$\mathrm{C}$ compares the complexity of various equalizer designs. 


\section{A. Synthetic data simulations}

1) Test transmission lines: The physical media for ADSL channels are metallic twisted pairs of wires, i.e. telephone lines. Commonly, transmission lines consist of several wire segments of different gauge and length. The gauge changes introduce an impedance mismatch and cause signal reflections. Most lines also contain bridged taps, open-circuited wire pairs bridged onto the main cable pair. Bridged taps are intended to offer flexibility for future alterations.

This paper uses a group of eight loops widely used in research simulations, called the carrier serving area (CSA) loops, which were proposed by Bell Systems in the early 1970s. Their impulse responses can be obtained via the LINEMOD software [78], which was developed based on two-port network transmission line theory [8, Sec. 3.5]. The simulations use the 8 CSA loops (available in [79]) in series with a $5^{t h}$ order Chebyshev Type I high-pass filter with cuton frequency at $4.8 \mathrm{KHz}$ and a high-pass filter with cut-on at $138 \mathrm{kHz}$, which serve to filter out the "plain old telephone system" (POTS) voiceband signal and the upstream signal, respectively.

2) Noise enviroment: Sources of DSL noise include impulse noise, consisting of impulses occurring at random times; background noise, modelled as additive white Gaussian noise (AWGN); and crosstalk. Crosstalk arises due to the fact that twisted pairs of wires are usually bundled together in large cables, and signals can leak from one cable to the next. Crosstalk is further divided into near-end crosstalk (NEXT) and far-end crosstalk (FEXT). NEXT tends to be dominant in ADSL transmissions [8]. It is generally modelled as a coupling filter fed by a white signal that has the same bandwidth and statistical properties as the modulated signal used by the adjacent loops. Our simulations use NEXT corresponding to 5 ISDN disturbers plus AWGN at $-140 \mathrm{dBm} / \mathrm{Hz}$ (relative to $23 \mathrm{dBm}$ input signal power).

3) Simulation results: Fig. 6 compares the bit rate of various designs. Delay optimization has been applied to all methods, and the TEQ length is 17 taps, which is a common choice in practice. Fig. 6(a) shows designs that are optimal in terms of some cost function other than the bit rate, Fig. 6(b) shows approximate or iterative methods, Fig. 6(c) shows methods that explicitly attempt to maximize the bit rate, and Fig. 6(d) shows the bit rate vs. delay for CSA loop 4.

The dual path TEQ computed the MMSE, MSSNR, and MDS TEQs, and picked the best one for one path, and then designed a Min-ISI TEQ optimized over a subset of tones for the second path. As such, it outperforms the other designs in Fig. 6(a). The approximations used in Fig. 6(b) usually induce a small loss in bit rate with respect to their counterparts in Fig. 6(a). The SAM algorithm seems to become stuck in local minima of the SAM cost function, which leads to a performance loss. The designs in Fig. 6(c) are listed in order of fewer approximations and more general structures, hence performance is expected to (and generally does) increase as we move left to right on the bar chart. In Fig. 6(d), the MDR, BMTEQ, TEQFB, and PTEQ do not require a full delay search in order to perform well, whereas the other designs are sensitive to the choice of delay and thus require a thorough delay search.

\section{B. AST data set}

Applied Signal Technology generously provided several measured ADSL data signals. The voltage signal from a telephone line was sampled at $2.5 \mathrm{MHz}$ and quantized. The signal was frequency-duplexed so that the upstream and downstream channels reside in two distinct frequency bands [8]. We resampled the data to $2.208 \mathrm{MHz}$ and used the C-REVERB2 training sequence, defined in [80, Sec. 10.4.5], to perform a (downstream) channel estimate for two recorded data sets. The left side of Fig. 7 shows the achievable bit rate for the 16 TEQ designs considered in the previous simulations, except now they have been used to equalize the two AST channels. Fig. 7 corroborates the results from the synthetic channels in Fig. 6.

\section{Complexity comparison}

The right side of Fig. 7 shows the approximate initialization complexity of the various equalizers. The FFT size is $N=$ 512 , the CP length is $\nu=32, \frac{1}{2} N+1$ tones are used, MERRY and SAM use 1000 iterations, the PTEQ was initialized via RLS, and the BM-TEQ and PTEQ use 100 iterations. As the designers of the MGSNR and MBR methods state that their methods are too complex for real time implementation, we do not show their (extremely high) implementation complexity. It is stressed that these figures are order of magnitude only, as the complexity of eigensolvers and other iterative methods depends greatly on the number of iterations needed for convergence. Except for the RLS initialization of the PTEQ, the complexity estimates were determined for the implementation of each design as it was originally presented. Complexity reduction techniques are beyond the scope of this paper.

If the original initialization of the PTEQ is used [18], the complexity is extremely high; but when the RLS initialization of [47] is used, the complexity is managable. The BM-TEQ, TEQFB, and PTEQ have high complexity, but they also have the highest performance. The MMSE and MERRY designs have very low complexity and average performance. The symmetric MMSE design has the lowest complexity, but it suffers a performance loss compared to the other designs. The high complexity and low bit rate of SAM suggests that its use be confined to time varying channels (for which it was designed).

\section{CONCLUSION}

This paper presented an overview of design methods for equalization in multicarrier systems where the channel delay spread is longer than the cyclic prefix. The majority of these techniques can be cast into a common formulation based on a maximization of a product of generalized Rayleigh quotients. In addition, we provided a unified notational framework and an extensive literature survey. One goal of this unification and common formulation was to clarify the subtle differences between these methods.

Based on the common formulation, several categories were distinguished, each leading to a different design strategy. A single generalized Rayleigh quotient led to suboptimal (in terms of bit rate maximization) single TEQ designs. On the 

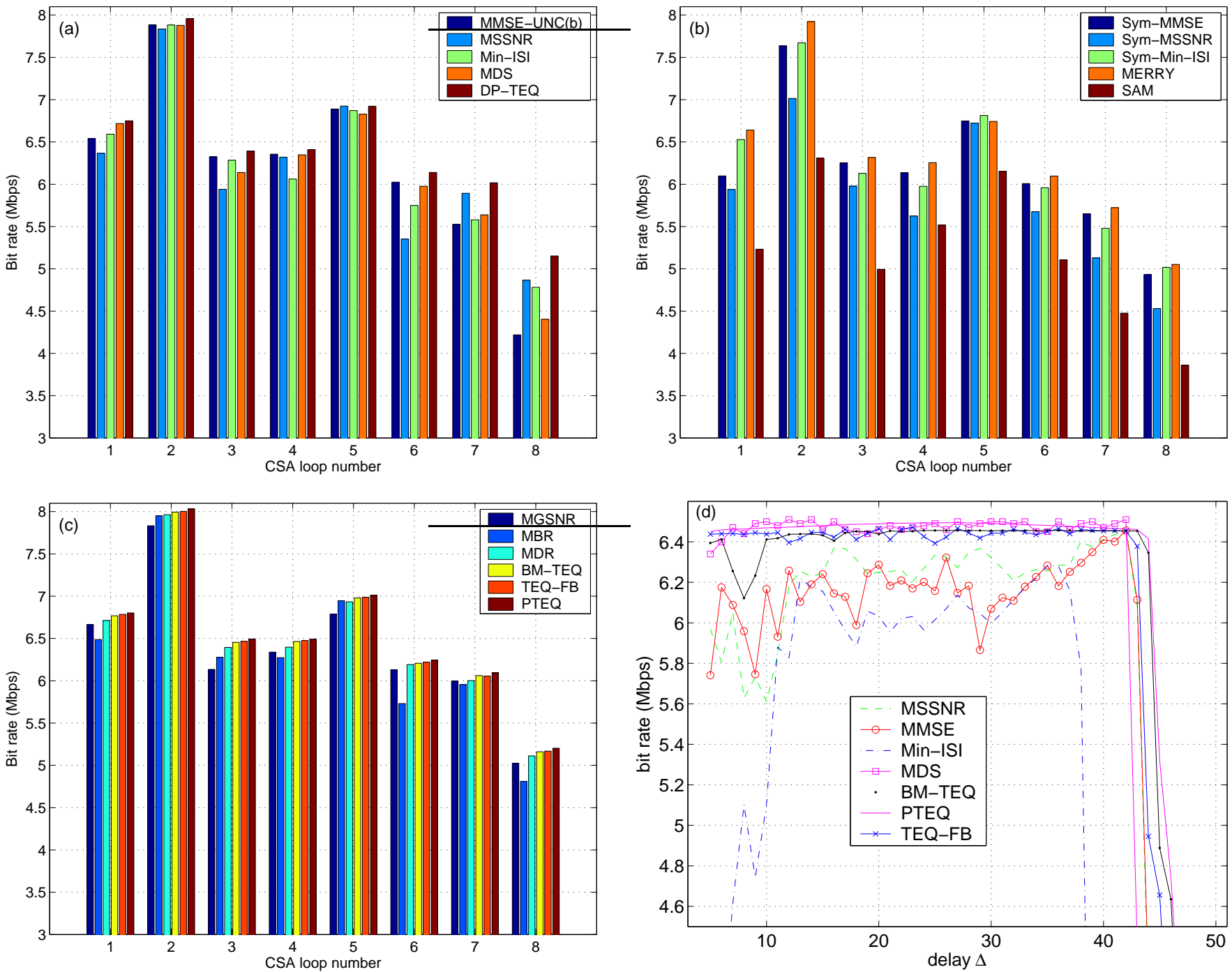

Fig. 6. Bit rate comparison. (a) designs that are optimal in terms of a measure other than the bit rate: minimum mean squared error (MMSE), maximum shortening SNR (MSSNR), minimum intersymbol interference (Min-ISI), minimum delay spread (MDS), and dual path TEQ. Left-to-right corresponds to top-to-bottom on the legend. (b) approximate or iterative designs: symmetric MMSE (Sym-MMSE), symmetric MSSNR (Sym-MSSNR), symmetric Min-ISI (Sym-Min-ISI), multicarrier equalization by restoration of reduncancy (MERRY), and sum-squared auto-correlation minimization (SAM). (c) designs that explicitly attempt to maximize the bit rate: maximum geometric SNR (MGSNR), maximum bit rate (MBR), maximum data rate (MDR), bitrate maximizing TEQ (BM-TEQ), TEQ filter bank (TEQFB), and per-tone equalization (PTEQ). (d) bit rate vs. delay for CSA loop 4.
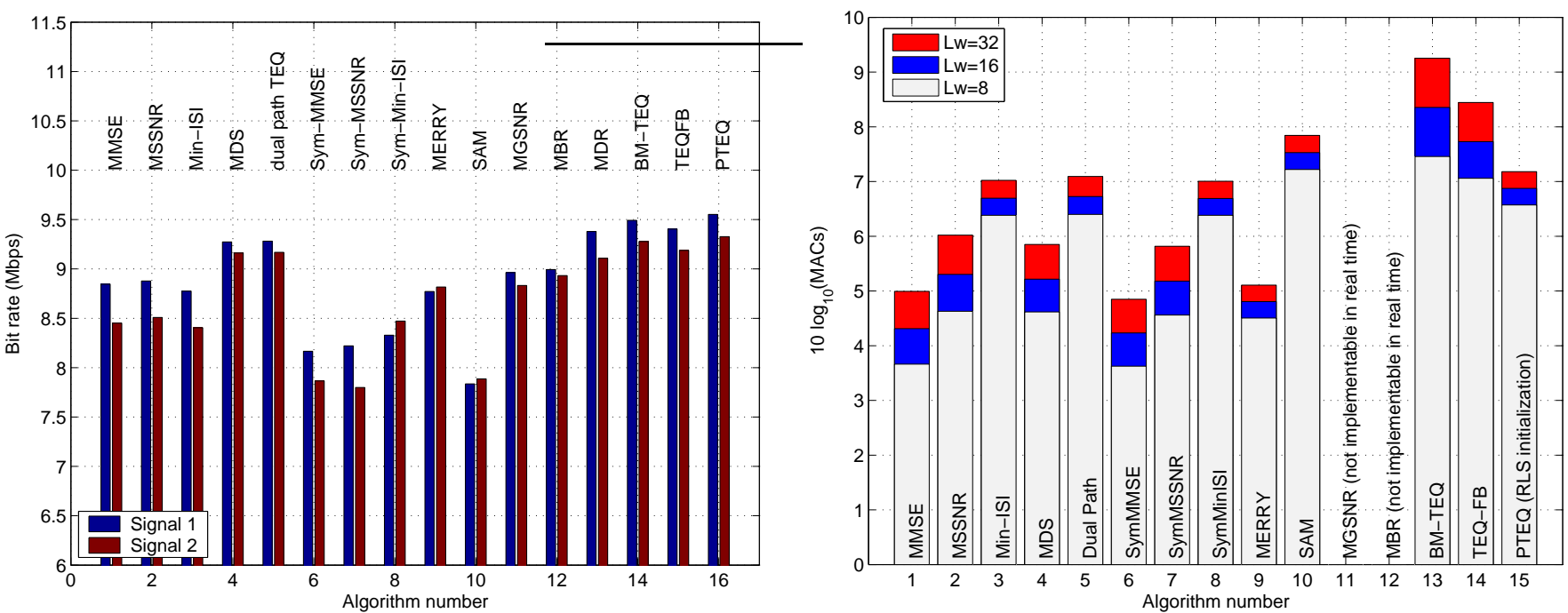

Fig. 7. Left: Bit rate acheived using the two measured AST channels. Right: Approximate implementation complexity. 
other hand, optimizing a single generalized Rayleigh quotient for each tone separately resulted in a bank of TEQ filters. The more difficult case with a product of generalized Rayleigh quotients is required for optimal single TEQ design. Intermediate designs could be devised based on multiple TEQ designs for subgroups of tones.

We have assessed computational complexity as well as bit rate performance using both synthetic and measured DSL channels. For ADSL channels, most designs yield bit rates that only differ by about $10 \%$. The literature appears to be moving towards the goal of perpetually increasing the bit rate.

\section{ACKNOWLEDGEMENTS}

The authors wish to thank John Treichler and Applied Signal Technology (Sunnyvale, CA) for providing the measured ADSL data, and Mike Woodhall (formerly at Applied Signal Technology) for answering our many questions about how the ADSL data was measured.

\section{REFERENCES}

[1] B. R. Saltzberg, "Performance of an Efficient Parallel Data Transmission System," IEEE Trans. on Comm. Technology, vol. 15, no. 6, pp. 805811, Dec. 1967.

[2] R. W. Chang, "Synthesis of Band-Limited Orthogonal Signals for Multichannel Data Transmission," Bell System Tech. Journal, vol. 45, pp. 1775-1796, Dec. 1966.

[3] S. B. Weinstein and Ebert P. M., "Data Transmission by FrequencyDivision Multiplexing Using the Discrete Fourier Transform," IEEE Trans. on Commun. Technology, vol. 19, no. 5, pp. 628-634, Oct. 1971.

[4] The European Telecomm. Standards Inst., "Radio Broadcasting System, Digital Audio Broadcasting (DAB) to Mobile, Portible, and Fixed Receivers," ETS 300 401, 1995-1997.

[5] The European Telecomm. Standards Inst., "Digital Video Broadcasting (DVB); Framing Structure, Channel Coding and Modulation for Digital Terrestrial Television," ETSI EN 300744 V1.4.1, 2001 Edition.

[6] The Inst. of Electrical and Electronics Engineers, "Wireless LAN Medium Access Control (MAC) and Physical Layer (PHY) Specifications. IEEE Std. 802.11a," 1999 Edition.

[7] The European Telecomm. Standards Inst., "Broadband Radio Access Networks (BRAN); High Performance Radio Local Area Networks (HIPERLAN) Type 2; System Overview,” ETR101 683 114, 1999.

[8] T. Starr, J.M. Cioffi, and P.J. Silvermann, Understanding Digital Subscriber Line Technology, Prentice Hall, 1999.

[9] J. A. C. Bingham, ADSL, VDSL, and Multicarrier Modulation, WileyInterscience, 2000

[10] P. J. Kyees, R. C. McConnell, and K. Sistanizadeh, "ADSL: A New Twisted-Pair Access to the Information Highway," IEEE Communications Magazine, vol. 33, no. 4, pp. 52-60, Apr. 1995.

[11] J. M. Cioffi, "A Multicarrier Primer," [Online]. Available: http://wwwisl.stanford.edu/people/cioffi/pdf/multicarrier.pdf.

[12] A. Peled and A. Ruiz, "Frequency Domain Data Transmission using Reduced Computational Complexity Algorithms," in Int. Conf. on Acoustics, Speech, and Signal Processing, Denver, Colorado, Apr. 1980, pp. $964-967$.

[13] J. A. C. Bingham, "Multicarrier Modulation for Data Transmission: An Idea Whose Time Has Come," IEEE Communications Magazine, vol. 28, no. 5, pp. 5-14, May 1990.

[14] Z. Wang and G. B. Giannakis, "Wireless Multicarrier Communications," IEEE Signal Proc. Mag., vol. 17, pp. 29-48, May 2000.

[15] T. Pollet, H. Steendam, and M. Moeneclaey, "Performance Degradation of Multi-Carrier Systems Caused by an Insufficient Guard Interval," in Proc. Int. Work. on Copper Wire Access Systems, Budapest, Hungary, Oct. 1997 , pp. $265-270$.

[16] J.-F. Van Kerckhove and P. Spruyt, "Adapted Optimization Criterion for FDM-based DMT-ADSL Equalization," in Proc. IEEE Int. Conf. on Comm., June 1996, vol. 3, pp. 1328-1334.

[17] M. Milosevic, L. F. C. Pessoa, B. L. Evans, and R. Baldick, "DMT Bit Rate Maximization with Optimal Time Domain Equalizer Filter Bank Architecture," in Proc. IEEE Asilomar Conf. on Signals, Systems, and Comp., Pacific Grove, CA, Nov. 2002, vol. 1, pp. 377-382.
[18] K. Van Acker, G. Leus, M. Moonen, O. van de Wiel, and T. Pollet, "Per Tone Equalization for DMT-Based Systems," IEEE Trans. on Comm., vol. 49, no. 1, pp. 109-119, Jan. 2001.

[19] L. Vandendorpe, "Fractionally Spaced Linear and DF MIMO Equalizers for Multitone Systems without Guard Time," Annals of Telecom., vol. 52, no. 1-2, pp. 21-30, Jan.-Feb. 1997.

[20] L. Vandendorpe, J. Louveaux, B. Maison, and A. Chevreuil, "About the Asymptotic Performance of MMSE MIMO DFE for Filter-Bank Based Multicarrier Transmission," IEEE Trans. on Comm., vol. 47, no. 10, pp. 1472-1475, Oct. 1999.

[21] T. Pollet, M. Peeters, M. Moonen, and L. Vandendorpe, "Equalization for DMT-Based Broadband Modems," IEEE Comm. Magazine, vol. 38, no. 5, pp. 106-113, May 2000.

[22] G. Leus and M. Moonen, "Per-Tone Equalization for MIMO OFDM Systems," IEEE Trans. on Signal Processing, Special Issue on Signal Processing for MIMO Wireless Comm. Systems, vol. 51, no. 11, pp. 2965-2975, Nov. 2003.

[23] G. Leus, I. Barhumi, and M. Moonen, "Per-Tone Equalization for MIMO-OFDM Systems," in Proc. IEEE Int. Conf. on Comm., May 2003, vol. 4, pp. 2345-2349.

[24] N. Al-Dhahir, "FIR Channel-Shortening Equalizers for MIMO ISI Channels," IEEE Trans. on Comm., vol. 49, no. 2, pp. 213-218, Feb. 2001.

[25] S. Barbarossa, G. Scutari, and A. Swami, "MUI-free CDMA Systems Incorporating Space-Time Coding and Channel Shortening," in Proc. IEEE Int. Conf. on Acoustics, Speech, and Signal Proc., May 2002, vol. 3, pp. 2213-2216.

[26] R. Samanta, R. W. Heath, Jr., and B. L. Evans, "Joint Space-Time Interference Cancellation and Channel Shortening," in Proc. IEEE Asilomar Conf. on Signals, Systems, and Computers, Pacific Grove, CA, Nov. 2003, vol. 1, pp. 32-36.

[27] T. Miyajima and Z. Ding, "Multicarrier Channel Shortening Based on Second-Order Output Statistics," in Proc. IEEE Workshop on Signal Proc. Advances in Wireless Comm., Rome, Italy, June 2003, pp. 623627.

[28] G. H. Golub and C. F. Van Loan, Matrix Computations, The Johns Hopkins University Press, Baltimore, MD, 1996.

[29] D. D. Falconer and F. R. Magee, "Adaptive Channel Memory Truncation for Maximum Likelihood Sequence Estimation,” Bell Sys. Tech. Journal, pp. 1541-1562, Nov. 1973.

[30] R. K. Martin, M. Ding, B. L. Evans, and C. R. Johnson, Jr., "Infinite Length Results and Design Implications for Time-Domain Equalizers," IEEE Trans. on Signal Processing, vol. 52, no. 1, pp. 297-301, Jan. 2004.

[31] P. J. W. Melsa, R. C. Younce, and C. E. Rohrs, "Impulse Response Shortening for Discrete Multitone Transceivers," IEEE Trans. on Comm., vol. 44, pp. 1662-1672, Dec. 1996.

[32] X. Yang, T. K. Sarkar, and E. Arvas, "A Survey of Conjugate Gradient Algorithms for Solution of Extreme Eigen-Problems of a Matrix," IEEE Trans. on Acoustics, Speech, and Signal Procesing, vol. 37, no. 10, pp. 1550-1556, Oct. 1989.

[33] R. K. Martin, J. Balakrishnan, W. A. Sethares, and C. R. Johnson, Jr., "A Blind, Adaptive TEQ for Multicarrier Systems," IEEE Signal Processing Letters, vol. 9, no. 11, pp. 341-343, Nov. 2002.

[34] M. Nafie and A. Gatherer, "Time-Domain Equalizer Training for ADSL," in Proc. IEEE Int. Conf. on Comm., Montreal, Canada, June 1997, vol. 2, pp. 1085-1089.

[35] G. Arslan, B. L. Evans, and S. Kiaei, "Equalization for Discrete Multitone Receivers To Maximize Bit Rate," IEEE Trans. on Signal Processing, vol. 49, no. 12, pp. 3123-3135, Dec. 2001.

[36] G. Arslan, Equalization Techniques for Multicarrier Modulation, Ph.D. thesis, The Univ. of Texas at Austin, Dec. 2000.

[37] K. Vanbleu, G. Ysebaert, G. Cuypers, M. Moonen, and K. Van Acker, "Bitrate Maximizing Time-Domain Equalizer Design for DMT-based Systems," IEEE Trans. on Comm., vol. 52, no. 6, pp. 871-876, June 2004.

[38] K. Vanbleu, G. Ysebaert, G. Cuypers, M. Moonen, and K. Van Acker, "Bitrate Maximizing Time-Domain Equalizer Design for DMT-based Systems," in Proc. IEEE Int. Conf. on Comm., May 2003, pp. 23602364.

[39] R. Freund and F. Jarre, "Solving the sum-of-ratios problem by an interior-point method," Tech. Rep., Bell Labs, 1999.

[40] S. Schaible, "Fractional Programming - a Recent Survey," J. Statistics and Management Systems, vol. 29, pp. 845-866, Mar. 2001.

[41] Y. Almogy and O. Levin, "A Class of Fractional Programming Problems," Operations Research, vol. 19, pp. 57-67, 1971. 
[42] N. Al-Dhahir and J. M. Cioffi, "Optimum Finite-Length Equalization for Multicarrier Transceivers," in Proc. IEEE Global Comm. Conf., San Francisco, CA, Nov. 1994, pp. 1884-1888.

[43] N. Al-Dhahir and J. M. Cioffi, "Optimum Finite-Length Equalization for Multicarrier Transceivers," IEEE Trans. on Comm., vol. 44, no. 1, pp. 56-64, Jan. 1996.

[44] N. Al-Dhahir and J. Cioffi, "A Band-optimized Reduced-complexity Equalized Multicarier Transceiver," IEEE Trans. on Comm., vol. 45, pp. 948-956, Aug. 1997.

[45] N. Lashkarian and S. Kiaei, "Optimum Equalization of Multicarrier Systems: A Unified Geometric Approach," IEEE Trans. on Comm., vol. 49, pp. 1762-1769, Oct. 2001.

[46] K. Vanbleu, G. Leus, and M. Moonen, "Per-Tone Equalization for DMTbased Transmission over IIR Channels," in Proc. IEEE Global Comm. Conf., 2001, pp. 405-409.

[47] K. Van Acker, G. Leus, M. Moonen, and T. Pollet, "RLS-Based Initialization for Per-Tone Equalizers in DMT Receivers," IEEE Trans. on Comm., vol. 51, no. 6, pp. 885-889, June 2003

[48] G. Ysebaert, M. Moonen, and T. Pollet, "Combined RLS-LMS Initialization for Per Tone Equalizers in DMT-receivers," in Proc. IEEE Int. Conf. on Acoustics Speech and Signal Processing, Orlando, FL, May 2002, pp. III-2537 - III-2540.

[49] K. Vanbleu, G. Ysebaert, G. Cuypers, and M. Moonen, "Bitrate Maximizing Per-Group Equalization for DMT-based Systems," Submitted to Elsevier Signal Processing, Aug. 2003.

[50] G. Goertzel, "An algorithm for the evaluation of finite trigonometric series," Amer. Math. Monthly, vol. 65, pp. 34-35, 1958

[51] J. S. Chow and J. M. Cioffi, "A Cost-Effective Maximum Likelihood Receiver for Multicarrier Systems," in Proc. IEEE Int. Conf. on Comm., June 1992, vol. 2, pp. 948-952.

[52] N. Al-Dhahir and J. M. Cioffi, "Efficiently Computed ReducedParameter Input-Aided MMSE Equalizers for ML Detection: A Unified Approach," IEEE Trans. on Info. Theory, vol. 42, no. 3, pp. 903-915, May 1996

[53] M. Van Bladel and M. Moeneclaey, "Time-Domain Equalization for Multicarrier Communication," in IEEE Global Telecomm. Conf., Nov. 1995, pp. 167-171.

[54] G. Ysebaert, K. Van Acker, M. Moonen, and B. De Moor, "Constraints in Channel Shortening Equalizer Design for DMT-based Systems," Signal Processing, vol. 83, pp. 641-648, Mar. 2003.

[55] K. Van Acker, Equalization and Echo Cancellation for DMT-based DSL Modems, Ph.D. thesis, Katholieke Universiteit Leuven, Jan. 2001, ftp://ftp.esat.kuleuven.ac.be/pub/sista/vanacker/reports/01-02.ps.gz.

[56] J. S. Chow, J. C. Tu, and J. M. Cioffi, "A Discrete Multitone Transceiver System for HDSL Applications," IEEE Journal on Selected Areas in Comm., vol. 9, no. 6, pp. 895-907, Aug. 1991.

[57] D. Pal, G. N. Iyengar, and J. M. Cioffi, "A New Method of Channel Shortening With Applications to Discrete Multi Tone (DMT) Systems," in Proc. IEEE Int. Conf. on Comm., June 1998, vol. 2, pp. 763-768.

[58] K. Van Acker, G. Leus, M. Moonen, S. Claes, and O. van de Wiel, "An improved optimization algorithm for time domain equalizer design in ADSL modems," in Proc. Int. Workshop on Copper Wire Access Systems, Oct. 1997, pp. 117-123.

[59] K. Van Acker, G. Leus, M. Moonen, S. Claes, and O. van de Wiel, "Improved Time Domain Equalization for ADSL," in Proc. ProRisc Work. on Circuits, Systems and Signal Processing, Mierlo, The Netherlands, Nov. 1997, pp. 615-620.

[60] C. Yin and G. Yue, "Optimal Impulse Response Shortening for Discrete Multitone Transceivers," IEE Electronics Letters, vol. 34, pp. 35-36, Jan. 1998.

[61] R. Schur, J. Speidel, and R. Angerbauer, "Reduction of Guard Interval by Impulse Compression for DMT Modulation on Twisted Pair Cables," in Proc. IEEE Global Comm. Conf., Nov. 2000, pp. 1632-1636.

[62] I. Ilani, "Time Domain Equalizer for DMT Transceivers - A Geometric Approach," US patent no. 6341298, Jan. 2002.

[63] R. K. Martin, J. Balakrishnan, W. A. Sethares, and C. R. Johnson, Jr., "Blind, Adaptive Channel Shortening for Multicarrier Systems," in Proc. IEEE Asilomar Conf. on Signals, Systems, and Computers, Pacific Grove, CA, Nov. 2002.

[64] D. Daly, C. Heneghan, and A. D. Fagan, "Minimum Mean-Squared Error Impulse Response Shortening for Discrete Multitone Transcievers," IEEE Trans. Signal Processing, vol. 52, no. 1, pp. 301-306, 2004.

[65] R. K. Martin, C. R. Johnson, Jr., M. Ding, and B. L. Evans, "Exploiting Symmetry in Channel Shortening Equalizers," in Proc. Int. Conf. on Acoustics, Speech, and Signal Proc., Hong Kong SAR, China, Apr. 2003, vol. 5, pp. V-97-V-100.
[66] R. K. Martin, M. Ding, B. L. Evans, and C. R. Johnson, Jr., "Efficient Channel Shortening Equalizer Design," EURASIP Journal on Applied Signal Processing, vol. 2003, no. 13, pp. 1279-1290, Dec. 2003.

[67] C. Ribeiro, V. Silva, and P. S. R. Diniz, "Linear Phase Impulse Response Shortening for xDSL DMT Modems," in IEEE Int. Telecom. Symp., Brazil, Sept. 2002, pp. 368-371.

[68] G. Arslan, B. L. Evans, and S. Kiaei, "Optimum Channel Shortening for Discrete Multitone Transceivers," in Proc. IEEE Int. Conf. on Acoustics, Speech, and Signal Proc., 2000, vol. 5, pp. 2965-2968.

[69] M. Ding, B. L. Evans, R. K. Martin, and C. R. Johnson, Jr., "Minimum Intersymbol Interference Methods for Time Domain Equalizer Design," in Proc. IEEE Global Comm. Conf., San Francisco, CA, Dec. 2003, vol. 4, pp. 2146-2150

[70] M. Ding, A. J. Redfern, and B. L. Evans, "A Dual-path TEQ Structure for DMT-ADSL Systems," in Proc. IEEE Int. Conf. on Acoustics, Speech, and Signal Proc., San Francisco, CA, May 2002, vol. 3, pp. 2573-2576.

[71] R. Schur and J. Speidel, "An Efficient Equalization Method to Minimize Delay Spread in OFDM/DMT Systems," in Proc. IEEE Int. Conf. on Comm., Helsinki, Finland, June 2001, vol. 5, pp. 1481-1485.

[72] S. Celebi, "Interblock Interference (IBI) Minimizing Time-Domain Equalizer (TEQ) for OFDM," IEEE Signal Processing Letters, vol. 10, no. 8, pp. 232-234, Aug. 2003.

[73] A. Tkacenko and P. P. Vaidyanathan, "Noise Optimized Eigenfilter Design of Time-domain Equalizers for DMT Systems," in Proc. IEEE Int. Conf. on Comm., New York, NY, Apr.-May 2002, vol. 1.

[74] M. de Courville, P. Duhamel, P. Madec, and J. Palicot, "Blind equalization of OFDM systems based on the minimization of a quadratic criterion," in Proc. IEEE Int. Conf. on Comm., Dallas, TX, June 1996, pp. 1318-1321.

[75] F. Romano and S. Barbarossa, "Non-Data Aided Adaptive Channel Shortening for Efficient Multi-Carrier Systems," in Proc. IEEE Int. Conf. on Acoustics, Speech and Signal Proc., Hong Kong SAR, China, Apr. 2003, vol. 4, pp. 233-236.

[76] W. Henkel and T. Kessler, "Maximizing the Channel Capacity of Multicarrier Transmission by Suitable Adaptation of the Time-domain Equalizer," IEEE Trans. on Comm., vol. 48, no. 12, pp. 2000-2004, Dec. 2000.

[77] J. Balakrishnan, R. K. Martin, and C. R. Johnson, Jr., "Blind, Adaptive Channel Shortening by Sum-squared Auto-correlation Minimization (SAM)," IEEE Trans. on Signal Processing, vol. 51, no. 12, pp. 30863093, Dec. 2003.

[78] D. G. Messerschmitt and A. Salvekar, "Linemod software for transmission line analysis," Stanford University. [Online]. Available: http://www.stanford.edu/ cioffi/linemod/linemod.html.

[79] G. Arslan, M. Ding, B. Lu, M. Milosevic, Z. Shen, and B. L. Evans, "MATLAB DMTTEQ Toolbox 3.1," The University of Texas at Austin, May 10, 2003. [Online.] Available: http://www.ece.utexas.edu/ bevans/projects/adsl/dmtteq/dmtteq.html

[80] International Telecommunications Union, "Asymmetrical Digital Subscriber Line (ADSL) Transceivers," ITU, 1999.

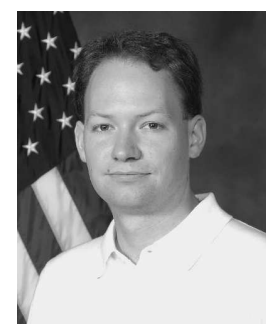

Richard K. Martin obtained dual B.S. degrees in physics and electrical engineering from the University of Maryland, College Park in 1999 (Summa Cum Laude), and an M.S. and a Ph.D. in electrical engineering from Cornell University in 2001 and 2004, respectively. Since August 2004, he has been an Assistant Professor at the Air Force Institute of Technology in Dayton, Ohio, where he is the signal processing curriculum chair. His research interests include equalization for multicarrier systems; blind, adaptive algorithms; reduced complexity equalizer design; and exploiting sparsity for performance improvement of adaptive filters. He has published seven journal papers, sixteen conference papers, and the book Theory and Design of Adaptive Filters Answer Book; and he has three patents pending. 


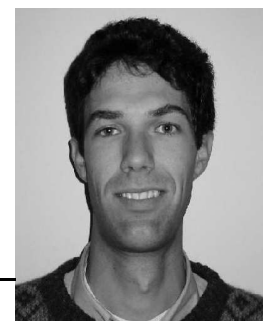

Koen Vanbleu was born in Bonheiden, Belgium, in 1976. In 1999 and 2004, he received the Master degree and Ph.D. degree in electrical engineering from the Katholieke Universiteit Leuven, Leuven, Belgium. From 1999 to 2003, he was supported by the Fonds voor Wetenschappelijk Onderzoek (FWO) Vlaanderen. Since November 2004, he is with the DSL Engineering division of Broadcom in Mechelen, Belgium. His research interests are in the area of digital signal processing for DSL communications.

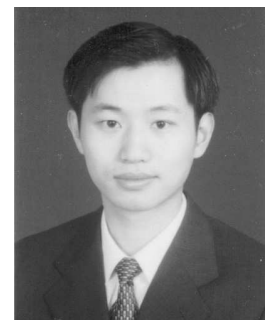

Ming Ding received his B.S. degree from Department of Electronic Science at Nankai University in 1995, M. ENG Degree from Department of Electrical and Computer Engineering at National University of Singapore in 1999, and Ph.D. Degree form Department of Electrical and Computer Engineering at The University of Texas at Austin in 2004, respectively. From 1995-1997, he was an R\&D engineer with the National Post \& Telecom. Industry Co., Shanghai, China. Form April 1999 to Aug. 2000, he worked as an R\&D Engineer for the Centre for Wireless Communications at the National University of Singapore. $\mathrm{He}$ is currently a member of Technical Staff with Bandspeed, Inc., Austin, Texas. His research interests include multicarrier modulation communications, adpative signal processing and VLSI prototyping of communication systems.

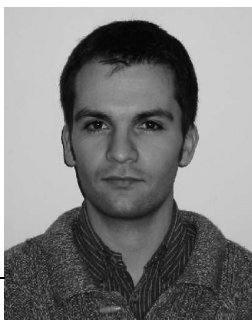

Geert Ysebaert was born in Leuven, Belgium, in 1976. He received the Master degree and Ph.D. degree in electrical engineering from the Katholieke Universiteit Leuven (KULeuven), Leuven, Belgium, in 1999 and 2004, respectively. From 1999 to 2003, he was supported by the Flemish Institute for Scientic and Technological Research in Industry (IWT). Since September 2004, he is with Alcatel, Research and Innovation in Antwerp, Belgium. His research interests are in the area of digital signal processing for DSL communications.

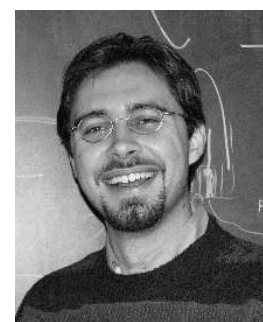

Milos Milosevic received his BS and MS in electrical engineering at the Illinois Institute of Technology in Chicago, IL in 1996 and 1998, respectively and his Ph.D. at the University of Texas at Austin in 2003. He was a member of engineering staff at the Motorola Semiconductor Product Sector and at the Texas Instruments Broadband Sector. He is currently a senior engineer in the Wireline Telemetry Department of Schlumberger in Houston, TX. His research interests are communication systems including multicarrier systems such as ADSL and

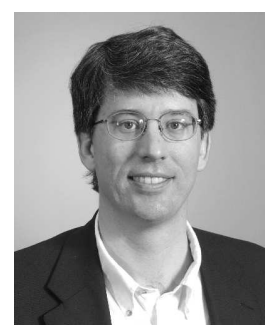

Brian L. Evans is a tenured Associate Professor in the Department of Electrical and Computer Engineering at The University of Texas at Austin. His BSEECS (1987) degree is from the Rose-Hulman Institute of Technology, and his MSEE (1988) and PhDEE (1993) degrees are from the Georgia Institute of Technology. From 1993 to 1996, he was a post-doctoral researcher at the University of California, Berkeley.

In signal processing, his research group is focused on the design and real-time software implementation of wireless OFDM basestations and ADSL transceivers for high-speed Internet access. His group developed the first time domain equalizer training method that maximizes a measure of bit rate and is realizable in real-time in fixedpoint software. In image processing, his group is focused on the design and real-time software implementation of high-quality halftoning for desktop printers and smart image acquisition for digital still cameras.

$\mathrm{He}$ is an Associate Editor for the IEEE Transactions on Signal Processing, a member of the Design and Implementation of Signal Processing Systems Technical Committee of the IEEE Signal Processing Society, and a Senior Member of the IEEE. He is the recipient of a 1997 US National Science Foundation CAREER Award.

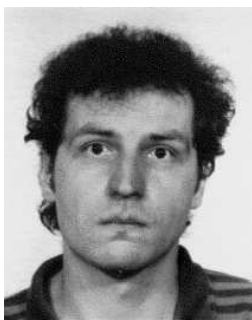

Marc Moonen received the Electrical Engineering degree and the Ph.D. degree in applied sciences from the Katholieke Universiteit Leuven, Leuven, Belgium, in 1986 and 1990, respectively. Since 2004, he is a Full Professor at the Electrical Engineering Department of Katholieke Universiteit Leuven, where he is currently heading a research team of $16 \mathrm{Ph} . \mathrm{D}$. candidates and postdocs, working in the area of signal processing for digital communications, wireless communications, DSL, and audio signal processing. He received the 1994 KU Leuven Research Council Award, the 1997 Alcatel Bell (Belgium) Award (with Piet Vandaele), and was a 1997 "Laureate of the Belgium Royal Academy of Science." He was the Chairman of the IEEE Benelux Signal Processing Chapter (19982002), and is currently a EURASIP Ad-Com Member (European Association for Signal, Speech and Image Processing, from 2000 to the present). He is Editor-inChief for the EURASIP Journal on Applied Signal Processing (from 2003 to the present), and a Member of the Editorial Board of Integration, the VLSI Journal, IEEE Transactions on Circuits and Systems II (2002-2003), EURASIP Journal onWireless Communications and Networking, and IEEE Signal Processing Magazine.

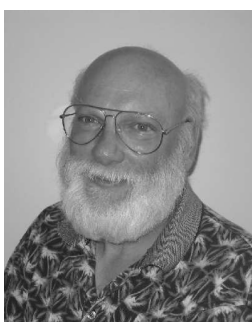

C. Richard Johnson, Jr. was born in Macon, GA in 1950. He received a B.E.E. with high honors from the Georgia Institute of Technology (1973); and an M.S.E.E. and a Ph.D. in E.E. with minors in Engineering-Economic Systems and Art History from Stanford University $(1975,1977)$. He is currently a Professor in the School of Electrical and Computer Engineering at Cornell University.

Dr. Johnson has received school, college, and national teaching awards, including selection by Eta Kappa $\mathrm{Nu}$ as the Outstanding Young Electrical Engineer in 1982 and as the C. Holmes MacDonald Outstanding Teacher among young professors of electrical engineering in the USA in 1983. Dr. Johnson was elected a Fellow of the IEEE in 1989 for "contributions to adaptive parameter estimation theory with applications in digital control and signal processing." In 1991, he was selected a Distinguished Lecturer of the Signal Processing Society of the IEEE. Dr. Johnson's current research interests are in adaptive parameter estimation theory that is useful in applications of digital signal processing to communications systems. 